\title{
On the transport, segregation and dispersion of heavy and light particles interacting with rising thermal plumes
}

\author{
Marcello Lappa ${ }^{1}$ \\ ${ }^{1}$ Department of Mechanical and Aerospace Engineering, University of Strathclyde, James Weir \\ Building, 75 Montrose Street, Glasgow, G1 1XJ, UK - email: marcello.lappa@strath.ac.uk
}

\begin{abstract}
:
A systematic numerical analysis is carried out on the multiplicity of patterns produced by inertial particles dispersed in a fluid and localized gravitational convection developing in the form of a rising thermal plume. In particular, specific numerical examples are presented to provide inputs for an increased understanding of the underlying flow-particle interaction mechanisms and cause-and-effect relationships. A rich spectrum of convective dynamics is obtained at the relatively high value of the considered Rayleigh number $\left(\mathrm{Ra}=10^{8}\right)$, which naturally allows the investigation of several intriguing effects (including, but not limited to, particle interaction with plume jet, associated vortices, shear instabilities and symmetry breaking phenomena). An important degree of freedom is introduced in the problem by changing the particle viscous drag through proper tuning of the related Stokes number (St). Similarly, inertia and weight of solid matter are varied parametrically by performing numerical simulations for both light and heavy particles at different values of the Froude number. This framework lets us identify the average behaviour of particles by revealing the mean evolution. We connect such statistics to the behaviour of the temporally evolving thermal plume, giving deeper insights into the particle transport mechanisms and associated dissipative dynamics.
\end{abstract}

Keywords: Buoyancy convection, Thermal Plumes, Inertial particles, settling, segregation, accumulation, clustering, trapping, pattern formation.

\section{Introduction}

The transport of an immiscible phase in a convectively unstable environment is relevant to many fields of applied physics as well as of industry. The problem underpins research in powder metallurgy, catalytic processes, mineral processing and nuclear re-processing. Indeed, in many of these fields, circumstances exist for which a material is in an initial liquid state (the so-called majority phase), which includes a second immiscible phase (the minority phase) in the form of dispersed solid particles or droplets requiring particular attention (see, e.g., Balboa-Usabiaga and Delgado-Buscalioni ${ }^{1}$; Ohta and Sussman ${ }^{2}$ ). Many materials with special features are produced in this way. Related examples include (but are not limited to) structural materials, many types of composites and alloys, electrical conductors, ceramics and magnetic substances (Zhao et al., ${ }^{3}$; Zhang et al., ${ }^{4}$; Markov'; Zhao et al., ${ }^{6}$ ). Usually, the presence of a gravity field forces the minority phase to experience buoyancy, i.e. the alloy components typically undergo rapid spatial separation through 
sedimentation or flotation. Superimposed on this is the fact that during these processes the melt is typically subject to a temperature difference. These gradients can be responsible for several additional complex and intriguing phenomena. In a gravity field, they lead in most cases to "natural" convective flows (thermogravitational convection).

This is just one example of circumstances in which the interplay between the motion of a dispersed phase under the influence of its own weight and convective flows of gravitational nature has to be seen as a significant aspect of the problem under investigation. Suspensions of dust, droplets, bubbles or other kinds of small particles in buoyant flows are widespread phenomena in nature and technology (Bürger and Wendland ${ }^{7}$ ). Additional relevance can be also found in typical industrial processes for the separation of particles from a suspension (the so-called size separation methods by which particles are extracted from a liquid according to their size). Such techniques use sedimentation as a natural means to filter out the solid phase. Accordingly, the controlled sedimentation of suspensions of small particles, is generally referred to as thickening or clarification depending on whether either the concentrated sediment or the clarified liquid (the majority phase) is considered as the main result of the process; in most situations, particles are separated while they are settling in a non-static medium (Parchevsky ${ }^{8,9}$ ).

In all such representative industrial instances, undesired motions of the medium can sometimes be mitigated; in general, however, fluid motion is significant and must be taken into account. This motion may have a preferred line of evolution in space, as in a pipe flow (see, e.g., Reeks and Skyrme ${ }^{10}$; Dasgupta et al., ${ }^{11}$ and references therein); in other cases, there is no preferred direction (e.g., homogeneous and isotropic turbulence, Maxey ${ }^{12}$; Wang and Maxey ${ }^{13}$ ).

Other relevant examples of convectively unstable fluids transporting fine dispersed particles include plumes from urban mass fires, the release in the atmosphere of smokes from industrial stacks (McGrattan et al., ${ }^{14}$ ), severe accidents in chemical or nuclear reactors (Kissane ${ }^{15}$ ), the fallout from nuclear testing (Moroz et al., ${ }^{16}$ ) and pyroclastic flows from volcanic eruptions (Valentine and Wohletz $^{17}$; Haszpra and Tél ${ }^{18}$ ).

As all these cases share a common factor, namely the existence of localized regions where the temperature is higher than that of the surroundings, in the present work we concentrate expressly on a fluid medium made unstable by the presence of buoyant thermal plumes.

Plumes are one of the most relevant examples in the natural, industrial or urban environment where lighter materials rise into an overlying fluid. Furthermore, plumes are not an exclusive prerogative of systems for which energy is provided to the fluid via a single source or a discrete distribution of sources of buoyancy. Indeed, thermal plumes can become a persistent feature of flow dynamics also when localized heating is replaced by homogeneous thermal boundary conditions. This typically occurs when the system is limited vertically by differentially heated solid walls and the fluid is in largely supercritical conditions [a theme generally known as turbulent Rayleigh-Bénard (RB) convection, see, e.g., Bucchignani and Stella ${ }^{19}$ ]. In such a context, thermal plumes can be regarded as the flow "ingredients" whose interrelations (or interactions) are responsible for the emergence of a well-defined "large scale" flow (see, e.g., Lappa ${ }^{20}$ and references therein). 
In general, thermal plumes have complex dynamics even if the considered fluid (e.g., a liquid) is assumed to be incompressible (Hier Majumder et al., ${ }^{21}$ ). The problem originating from their interaction with a dispersed solid (or immiscible) phase, however, can be made even more complex by the peculiar properties of the particle velocities which tend to behave as a compressible velocity field. Even though the considered fluid flow is incompressible, inertia causing compressibility effects in the particle velocity field, can produce depletion of dispersed solid matter in some regions, and its accumulation in others (such events, frequently referred to as preferential concentration, are associated with the presence of regions with either extremely high or low particle concentrations ${ }^{22,23}$ ). In such a context, numerous studies have highlighted the significant role played in such dynamics by the interplay between the just mentioned compressibility affecting the particle velocity field and the specific amount of vorticity possessed by the carrier fluid flow ( $\operatorname{Re}^{24}$ and references therein). As instructive and specific examples, the reader may consider the works by Maxey et al., ${ }^{25}$ and Saw et al., ${ }^{26}$ where numerical and experimental evidence was clearly reported, respectively, that dense particles can undergo remarkable segregation phenomena in high-Reynolds-number homogenous isotropic turbulence. Similarly, Boffetta and De Lillo ${ }^{27}$ could numerically predict the emergence of network-like regions of high particle density in turbulent flows. Along these lines, there is widespread consensus nowadays that such remarkable phenomena of preferential concentration depend on the ability of the various eddies present in the considered flow to act as "centrifuges" ejecting particles having density larger than that of the fluid and entrapping them in the opposite case. Compressibility effects are introduced because such vortices create a positive or negative divergence in the particle velocity field according to the relative density of dispersed matter with respect to the surrounding fluid (Squires and Eaton, ${ }^{28}$; Biferale et al., ${ }^{29}$; Bec et al., ${ }^{30}$ ). As a result, heavy particles tend to accumulate at the boundaries of vortices (the regions with small velocity and large shear rate), while it is vice-versa in the case of light particles.

Some initial ideas along these lines were already present in the works by Eaton and Fessler ${ }^{31}$ and Raju and Meiburg ${ }^{32}$ where the tendency of heavy inertial particles to leave the neighbourhood of a vortex was identified as the main mechanism by which vortical structures cause concentration nonuniformities in typical technological processes. These mechanisms can obviously interfere with the sedimentation process.

Historically, this subject took a peculiar path essentially depending on the widespread practice to develop theoretical models based on the interaction of particles with isothermal two-dimensional cellular flow fields consisting of vortices with well-known analytic representation (chosen as an idealized view of effective processes or to mimic turbulent flows, and supported by the need to study the problem in well-controlled and deterministic conditions). A milestone work, on which several numerical approaches would successively rely, was the analysis by Stommel ${ }^{33}$. This author was the first to provide a description of how heavy particles can be permanently suspended in a steady twodimensional array of vortices only if their inertia is neglected. Later Maxey and Corrsin ${ }^{34}$ demonstrated that inertia, though weak, eventually causes all particles to settle out. 
Some additional useful (general) information about these subjects can be found in the book by Clift et al., ${ }^{35}$ and in the aforementioned work by Burger and Wendland ${ }^{6}$. While in the former a variety of data have been provided about the typical drag coefficient of a sedimenting particle depending on its shape and Reynolds number, the latter presents a review of the so-called Kynch's kinematic sedimentation theory, and its extension to different circumstances (continuous sedimentation, flocculent and polydisperse suspensions, vessels with varying cross-section, centrifuges and several space dimensions).

Though, owing to page limits we do not strive to review all aspects relating to the sedimentation of a dispersed particle in a quiescent or non-quiescent medium, we wish to mention here also the excellent analyses by Raju and Meiburg ${ }^{32,36}$ (from which we took most of the inspiration at the root of the present work), Dávila and Hunt ${ }^{37}$, Eames and Gilbertson ${ }^{38}$, Chen et al., ${ }^{39}$, Ravichandran et al., ${ }^{40}$ and Bergougnoux et al., ${ }^{41}$. Wang and Maxey ${ }^{13}$ and Dávila and Hunt ${ }^{37}$ demonstrated that the transient nature of turbulence can give rise to increased settling speeds for sufficiently small particles. Good et al., ${ }^{42}$ found that the reduced settling speeds of relatively large particles (see, e.g., Kawanisi and Shiozaki ${ }^{43}$ ) are due to nonlinear drag effects. They also demonstrated that falling particles are more responsive to vertical than to horizontal fluctuations.

Works addressing particles interacting with non-isothermal conditions and thermal convection, however, are much more rare and sparse. Akbar et al., ${ }^{44}$ and Puragliesi et al., ${ }^{45}$ considered an airfilled differentially heated cavity with horizontal temperature gradient for the case in which the temperature gradient is horizontal and the flow develops accordingly a stable thermal stratification in the centre of the cavity. While the former concentrated on Rayleigh numbers in the range of $10^{2}$ to $8 \times 10^{5}$ and particles with size $1 \mu \mathrm{m}, 200 \mathrm{~nm}$, and $50 \mathrm{~nm}$, the latter focused on particles with diameter $15,25,35 \mu \mathrm{m}$ and $\mathrm{Ra}=10^{9}$ and $10^{10}$. These studies provided evidence that some conditions can be identified (in terms of particle size and Rayleigh number), for which near-equilibrium particle recirculation zones are maintained by thermal convection (particles being allowed to remain suspended in these zones). Along the same lines, Puragliesi et al., ${ }^{45}$ highlighted that strong recirculating zones created in proximity to the heated wall might contribute to decrease the average rate of gravitational settling by temporarily lifting and segregating particles.

These mechanism are made particularly complex by the fact that, in addition to being compressible, just as thermal convection (see, e.g., Lappa ${ }^{46}$ ), particle dynamics are, in general, dissipative.

Continuing our computational investigation (see, e.g., Refs ${ }^{47-53}$ ) into this general area, in the present study (unlike earlier works, which considered synthetic flows, isotropic turbulence or other circumstances) we target expressly the case of particles interacting with thermal plumes in thermogravitational flow. An increased understanding of these processes (see, e.g., Gan et al., ${ }^{54}$; Akbar et al., ${ }^{44}$; Puragliesi et al, ${ }^{45}$; Haeri and Shrimpton ${ }^{55}$ ) is likely to produce important benefits or advantages, for example, better process control, increased efficiency, improved reproducibility and the ability to modify or devise products for innovative applications in chemistry, physics and biomaterials. 


\section{Mathematical Models and Methods}

\subsection{Fluid, geometry and boundary conditions}

We consider a portion of liquid limited by two horizontal walls placed $2 \mathrm{~L}$ apart, as shown in Figure 1 and circumstances for which the temperature field permits the formation of a localized thermal plume.

A single laminar plume, generally known in the literature as a unbounded or "free" plume is produced when the fluid located in proximity to a point or horizontal line heat source becomes lighter and starts to rise (as an example, a two-dimensional line source plume can be obtained if a horizontal heated wire is immersed in a box of cold fluid, see, e.g., the experiments of Rouse et al., 56 .

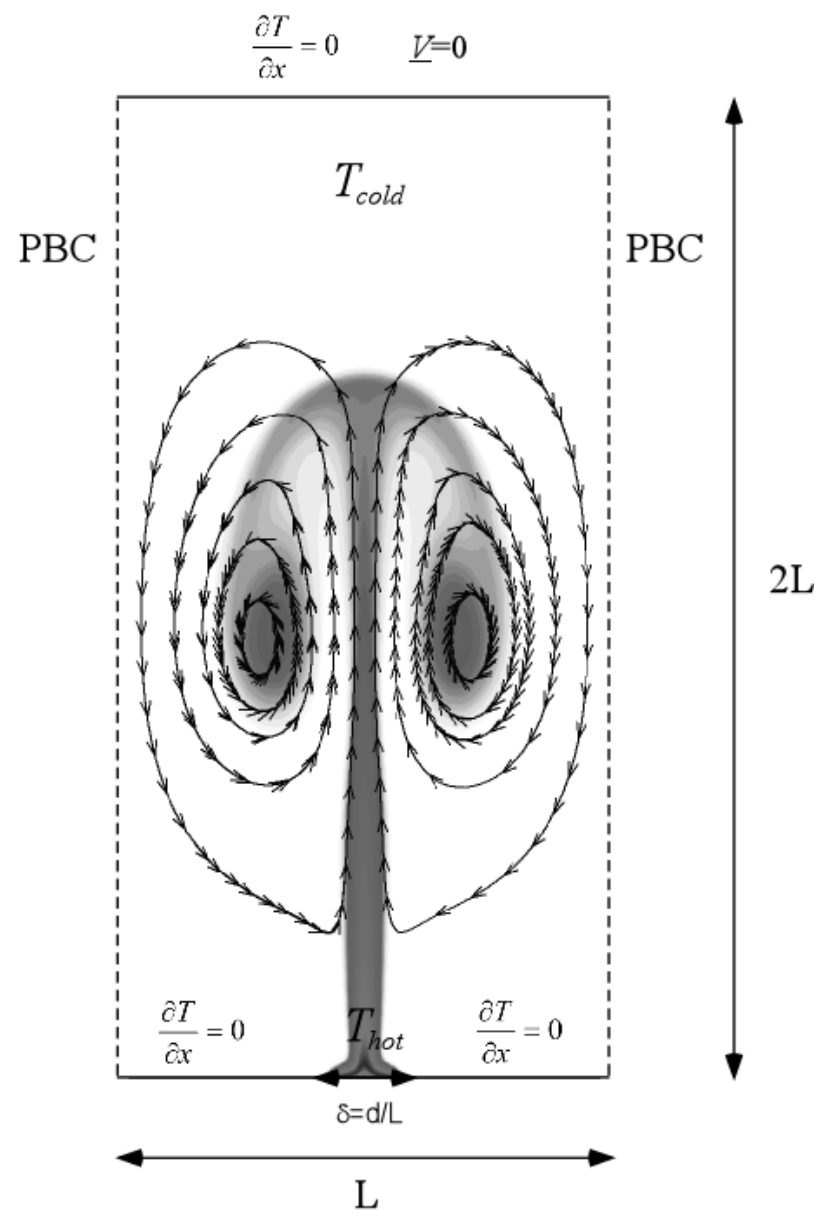

Figure 1: Physical domain and boundary conditions

The theory for steady laminar plumes is in a mature state. According to scaling arguments, the vertical velocity can be considered constant (Batchelor ${ }^{57}$ ). Asymptotic analyses valid for the plume stem far from the leading edge (the cap) are due to Gebhart et al., ${ }^{58}$, Worster ${ }^{59}$ and Liñán and Kurdyumov $^{60}$. Other authors (e.g., Moses et al., ${ }^{61}$; Kaminski and Jaupart ${ }^{62}$ ) have specifically 
concentrated on the behaviour of the plume cap (not considered by asymptotic analyses). Numerical results are available for different values of the Prandtl number (e.g. Fujii ${ }^{63}$; Brand and Lahey ${ }^{64}$; Worster $^{59}$; Hier Majumder et al., ${ }^{21}$; Hernandez ${ }^{65}$ ).

In particular, Hier Majumder et al., ${ }^{21}$ considered values of the Prandtl numbers spanning the interval from $10^{-2}$ up to $10^{4}$, identifying the existence of four different regimes of plume development. Following their work, here we focus on the case of plumes in the so-called IVND (inviscidnondiffusive) regime. The reason of such a choice resides essentially in the fact that this is the most turbulent of the four regimes, thus being representative of many effective situations of practical and technological interest.

The plume is assumed to be produced by a heat source of finite length $d$ located on the bottom of the physical domain. The heat source is modelled assuming there a constant temperature $\mathrm{T}_{\text {hot }}$ while the rest of the bottom wall and the entire top wall are adiabatic. No slip conditions are set for velocity on such walls (confined plume), while periodic boundary conditions (PBC) are considered for velocity and temperature fields at the two lateral boundaries (placed L apart, as shown in Fig. 1).

At the initial instant $\mathrm{t}=0$ the fluid is assumed to be in quiescent conditions and at a uniform temperature $\mathrm{T}_{\text {cold }}$.

Hereafter, we indicate with $\Delta \mathrm{T}$ the temperature difference between the temperature of the heat source and the temperature of the liquid at $\mathrm{t}=0$, i.e. $\Delta \mathrm{T}=\mathrm{T}_{\text {hot }}-\mathrm{T}_{\text {cold. }}$. Moreover, we denote by $\alpha$ the fluid thermal diffusivity, $\rho_{\mathrm{F}}$ the fluid density, $\nu$ the fluid kinematic viscosity and $\mu$ the dynamic viscosity. This leads us to introduce more formally the various dimensionless parameters involved in the problem. At least three independent dimensionless parameters have to be specified

$$
\begin{aligned}
& \operatorname{Pr}=\frac{v}{\alpha} \\
& \operatorname{Ra}=\frac{g \beta_{T} \Delta T L^{3}}{v \alpha} \\
& \operatorname{Fr}=\frac{v}{L^{3 / 2} \sqrt{g}}
\end{aligned}
$$

known as the Prandtl, Rayleigh and Froude numbers, respectively (where $\mathrm{g}$ is the gravity acceleration and $\beta_{\mathrm{T}}$ is the thermal expansion coefficient).

The ranges of existence of the aforementioned IVND regime are $\operatorname{Ra} \geq \mathrm{O}\left(10^{8}\right)$ for $\operatorname{Pr}=10^{-3}, \operatorname{Ra} \geq \mathrm{O}\left(10^{6}\right)$ for $\operatorname{Pr}=\mathrm{O}(1), \mathrm{Ra} \geq \mathrm{O}\left(10^{7}\right)$ for $\mathrm{Pr}>\mathrm{O}\left(10^{4}\right)$.

Accordingly, we select $\operatorname{Pr}=8\left(\mathrm{NaNO}_{3}\right.$, see Table I) and $\mathrm{Ra}=10^{8}$. 
TABLE I: Liquid properties

\begin{tabular}{ll}
\hline Fluid $\left(\mathrm{NaNO}_{3}\right)$ density $\rho, \mathrm{kg} \mathrm{m}^{-3}$ & 1904 \\
Kinematic viscosity $\mathrm{v}, \mathrm{m}^{2} \mathrm{~s}^{-1}$ & $1.27 \times 10^{-6}$ \\
Thermal diffusivity $\alpha, \mathrm{m}^{2} \mathrm{~s}^{-1}$ & $1.58 \times 10^{-7}$ \\
Thermal expansion coefficient $\beta_{\mathrm{T}}, \mathrm{K}^{-1}$ & $1.25 \times 10^{-3}$
\end{tabular}

\subsection{Nondimensional balance equations}

The non-dimensional balance equations governing fluid motion can be obtained from scaling the coordinates by $L$ and the velocity components by the energy diffusion velocity $V_{\alpha}=\alpha / L$; related scales for time and pressure are, respectively, $\mathrm{L}^{2} / \alpha$ and $\rho_{\mathrm{F}} \alpha^{2} / \mathrm{L}^{2}$. The temperature is measured with respect to its initial value $\mathrm{T}_{\text {cold }}$ and is made nondimensional as $\left(\mathrm{T}-\mathrm{T}_{\text {cold }}\right) / \Delta \mathrm{T}$.

In such a framework and using the Boussinesq approximation, the continuity and momentum equations simply read:

$\underline{\nabla} \cdot \underline{\mathrm{V}}=0$

$\frac{\partial \underline{V}}{\partial}=-\underline{\nabla} p-\underline{\nabla} \cdot[\underline{V} \underline{V}]+\operatorname{Pr} \nabla^{2} \underline{V}+\operatorname{Pr} \operatorname{RaT} \underline{\hat{n}}$

where $\underline{V}$ and $\mathrm{p}$ are the fluid velocity and pressure, respectively, and $\underline{\underline{h}}$ is the unit vector along the direction of gravity.

These equations must be supplemented with the energy balance equation, which in nondimensional form reads:

$$
\frac{\partial T}{\partial t}+\underline{\nabla} \cdot[\underline{V T}]=\nabla^{2} T
$$

\subsection{Solid matter}

Dispersed impurities or particles have a finite size and typically a mass density different from that of the carrier fluid, which makes a representation based on the concepts of point-like particles with negligible mass, and moving with a velocity that inherits the divergence-free property of the carrier flow, a really oversimplified and inaccurate model. A relevant and complete mathematical framework must take into account their inertia.

In other words, this means that accounting for the dispersed phase is equivalent to introducing additional degrees of freedom in the considered problem (particle shape, size and density). Related dimensional parameters are the particle density $\rho_{\mathrm{P}}=\left(\xi \rho_{\mathrm{F}}\right)$ (where $\xi$ is the ratio of the solid matter to 
the fluid density), and the particle radius $\widetilde{R}$ (we assume particles to be perfectly spherical). Another relevant dimensional quantity is the so-called particle relaxation (or aerodynamic response) time $(\tau)$, which in the literature is generally defined as:

$\tau_{\text {part }}=\frac{2}{9} \frac{\widetilde{R}^{2}}{v}$

For the sake of consistency with earlier works addressing companion subjects ${ }^{47-53}$, as the characteristic time scale of the fluid flow, we consider:

$\tau_{\text {flow }}=\frac{L^{2}}{v}$

Moreover, to properly account for the effect of gravity on particle motion, we introduce a third relevant time scale into the problem, namely, the particle setting time:

$\tau_{\text {sett }}=\frac{9}{2} \frac{v L}{g \widetilde{R}^{2}}$

This quantity relates to the amount of time that a particle falling (or rising) with its terminal velocity would take to cover a distance equal to the height of the cavity.

The fundamental difference between this time scale and $\tau_{\text {flow }}$ can be easily understood just taking into account that, while $\tau_{\text {flow }}$ is directly connected to the time required by the particle to respond to unsteady forcing eventually caused by fluctuating velocities of the carrier flow, $\tau_{\text {sett }}$ should be regarded as a time scale linked to the forcing of steady nature that gravity exerts directly on particles (this being dependent, in turn, solely on gravity acceleration, particle size and fluid viscosity).

In practice, additional insights into this problem can be obtained by introducing specific nondimensional parameters measuring the relative importance of the characteristic times so far introduced. Such nondimensional ratios can be defined as:

$\frac{\tau_{\text {part }}}{\tau_{\text {flow }}}=\frac{2}{9} \frac{\widetilde{R}^{2}}{L^{2}}=S t$

generally known as the Stokes number, and:

$\frac{\tau_{\text {sett }}}{\tau_{\text {part }}}=\left(\frac{9}{2}\right)^{2} \frac{L v^{2}}{g \widetilde{R}^{4}}=\frac{F r^{2}}{S t^{2}}$ 
$\frac{\tau_{\text {sett }}}{\tau_{\text {flow }}}=\left(\frac{9}{2}\right) \frac{v^{2}}{g L \widetilde{R}^{2}}=\frac{F r^{2}}{S t}$

which, as shown by (Raju and Meiburg ${ }^{32}$ ) can be also written in combined form as :

$\sqrt{\frac{\tau_{\text {sett }}}{\tau_{\text {flow }}} \frac{\tau_{\text {part }}}{\tau_{\text {flow }}}}=\frac{v}{L^{3 / 2} \sqrt{g}}=F r$

which indicates that the Froude number can be linked directly to the square root of the product of the two time scale ratios in eqs. (8b) and (8c), respectively.

Given such premises, the limit $\mathrm{St} \rightarrow 0$ can be seen as the condition for which a fixed viewer would see the particle moving with the same velocity as the carrier flow, i.e. particles "frozen" in the natural convective field (in other words, particles simply moving with the local fluid velocity as ideal mass-less and size-less Lagrangian tracers), the opposite limit St $>>1$, representing situations in which particles would be prevented from responding to any velocity change in such a flow due to excessive inertia.

Similarly, the other two nondimensional ratios can be used in principle to characterize the "residence time" of the particle in the considered domain, i.e. the time interval during which the surrounding flow can affect a particle in order to enhance or delay its settling (and create accordingly nonuniformities in an initially uniform dispersion of solid matter). In such a context, therefore, the limit $\mathrm{Fr} \rightarrow 0$ may be interpreted as the ideal condition that would be attained by a particle undergoing unperturbed gravitational (purely vertical) motion (all particles moving in synchronized manner, no non-uniformities in the particle concentration field being allowed).

Moreover, with the above mélange of characteristic numbers the nondimensional particle terminal velocity can be cast in compact form as:

$V_{T}=\frac{2}{9} \widetilde{R}^{2} L \frac{g(\xi-1)}{v \alpha}=\frac{\gamma S t}{\operatorname{Pr}}(\xi-1)=\frac{\operatorname{Pr}}{F r^{2}} S t(\xi-1)$

which indicates that for a fixed fluid ( $\mathrm{Pr})$ and container height $(\mathrm{Fr})$, such a nondimensional velocity depends only on the Stokes number (St) and particle density (via $\xi$ ).

Let us recall that this quantity represents the ideal speed that a particle would attain asymptotically (after an initial transient time required to establish a perfect balance between gravity and viscous drag) while falling in a still (motionless) fluid.

Evaluation of the effective particle velocity in a convectively unstable medium requires obviously a dedicated numerical approach and the solution of an additional set of equations, whose nature and number depends on the specific strategy undertaken to capture the motion of the dispersed solid phase. Indeed, many valuable methods have been elaborated over the years to deal with such problems. These techniques, which have enjoyed a varying degree of use and success depending on 
the specific problem considered, can roughly be categorized in methods entirely based on the assumption or concept of "continuum" (where particles are globally treated as a "fluid" by assuming that the carrier fluid and the set of particles behave as interpenetrating continua) or partially involving a discrete representation of reality (with particles being monitored and tracked as separate entities with respect to the background fluid flow). Each method, of course, has its own convenience and bottlenecks. The former allow particle diffusion to be incorporated directly into the model through the introduction of some "production terms" in the momentum and energy equations. As illustrated by many authors (see, e.g., Marble ${ }^{66}$; Dasgupta et al., ${ }^{11}$; De' Michieli Vitturi et al., ${ }^{67}$ ), these techniques work particularly well for the case of particles in gases for which the adoption of an "equivalent gas model" is used to treat the gas-particle mixture as a homogeneous medium (with such methods the treatment of the dispersed phase is particularly convenient and efficient as, in place of being considered as separate entities, particles and their local density are accounted for by solving a conservation equation formulated for the dispersed phase in terms of particle concentration).

As we are specifically interested in the "patterning behaviours" produced by particles, we base the present simulations on a hybrid approach in which particles are tracked via the solution of a specific Lagrangian equation. This gives us the possibility to track every single particle as a separate entity and identify the emerging "ensemble" phenomena through the analysis of the related collective behaviour. Under the assumption $\mathrm{St}<<1$, such equation, known as the Maxey-Riley equation, can be written (using the present reference quantities) in condensed form as:

$\frac{d \underline{V}_{\text {partcl }}}{d t}=\frac{1}{\xi+1 / 2}\left[-\frac{\operatorname{Pr}}{S t}\left(\underline{V}_{\text {partcl }}-\underline{V}\right)+\frac{3}{2} \frac{\partial \underline{V}}{\partial t}+\frac{3}{2}(\underline{V} \cdot \underline{\nabla} \underline{V})\right]+\frac{\xi-1}{\xi+1 / 2} \gamma \underline{\underline{n}}$

where $\underline{V}_{\text {part }}\left(u_{\text {part }}, v_{\text {part }}, w_{\text {part }}\right)$ is the particle velocity and $\mathrm{d} \underline{V}_{\text {part }} / \mathrm{dt}$ is its time derivative. This equation includes the viscous drag forces (first term at the right hand side), the added mass effect relating to the material derivative of the fluid velocity (second and third terms) and the effect of gravity (fourth term) depending on the parameter $\gamma$, which, in turn, reads

$\gamma=\frac{g L^{3}}{\alpha^{2}}=\left(\frac{\mathrm{Pr}}{F r}\right)^{2}$

( $\gamma$ may be also regarded as an analogue of the so-called Galileo number defined as $\mathrm{Ga}=\mathrm{gL} / \mathrm{v}^{2}=\gamma / \mathrm{Pr}^{2}$ ). Such well-known equation, originally introduced by Maxey and Riley ${ }^{68}$ (since then it has enjoyed a widespread use in the literature, see, e.g., Sapsis and Haller ${ }^{69}$; Kuhlmann et al., ${ }^{70}$ and references in these papers), is based on the so-called dilute-system assumption, i.e. particle motion cannot alter the liquid flow (the reader being referred to other works in the literature, such as Dasgupta et al., ${ }^{11}$, for the opposite situation in which the mass loading of particles is so high that two-way coupling numerical approaches must be necessarily used). Moreover, we assume particles to be independent of each other. Despite such limitations, as also illustrated by Akbar et al., ${ }^{44}$ and Sapsis and Haller ${ }^{69}$, this 
equation has proved of fundamental importance in the definition and elaboration of criteria to predict the inertial particle relocation/clustering dynamics in in a variety of flows and conditions of practical interest and relevance. We do not consider thermophoretic forces and Brownian motion effects as they are expected to be negligible for the conditions considered in the present work (Puragliesi et al., $\left.{ }^{45}\right)$.

\subsection{Solution Method and Mesh Refinement Study}

Balance equations (2-4) have been solved by a time-explicit finite-difference projection (primitivevariable) method. The method has been implemented with a staggered grid arrangement, deriving velocities and pressure in a segregated manner. A synthetic description of the related approach can be provided as follows.

The momentum equation is initially integrated with respect to time by disregarding the influence of pressure. In this way, an intermediate velocity field is determined that has no precise physical meaning, but has some "mathematical relevance" as it possesses the same amount of vorticity that the physically relevant velocity field should have. The equation required for this step reads:

$\underline{V}^{*}=\underline{V}^{n}+\Delta t\left[-\underline{\nabla} \cdot(\underline{V} \underline{V})+\operatorname{Pr} \nabla^{2} \underline{V}\right]^{n}+\operatorname{Pr} \operatorname{RaT}^{n} \underline{\hat{n}}$

The pseudo-velocity field obtained in this way does not satisfy the incompressibility constraint and must, therefore, be corrected. This is achieved by reintroducing the pressure gradient previously disregarded as follows:

$\underline{V}^{n+1}=\underline{V}^{*}-\Delta t \underline{\nabla} p^{n}$

As the pressure $\mathrm{p}$ appearing in this equation plays the role of an unknown, an additional equation is needed for its determination. Such equation, explicitly needed for the problem mathematical closure, is obtained by substituting the above expression for the velocity at the time step $n+1$ into the continuity equation:

$\nabla^{2} p^{n}=\frac{1}{\Delta t} \underline{\nabla} \cdot \underline{V}^{*}$

With this modus operandi, the method ensures that the final velocity field $\underline{V}^{n+1}$ (eq. (12b) is solenoidal (as required for an incompressible flow) and possesses the same vorticity that would satisfy the vorticity balance equation (separate equation obtained by applying the curl operator to the momentum equation). The resulting field is, therefore, consistent with the physics of the considered problem and "unique" from a mathematical point of view (by virtue of the so-called 
Ladyzhenskaya $^{71}$ decomposition theorem, which states that the uniqueness of a vector field is guaranteed when its divergence and curl are given).

The accuracy of such a method obviously depends on the nature and order of the schemes used to integrate effectively the above equations and the related time and space integrations steps.

For the present work, in particular, forward differences have been used to discretize the time derivatives, coupled with central-differencing schemes in space (second order accurate) for the momentum equation and upwind schemes (third-order accurate) for the energy equation (the partially different treatment of the energy equation has been motivated by the need to resolve with sufficient accuracy the strong temperature gradients established between the plume and the surrounding fluid). With regard to the spatial integration steps, the sensitivity of the overall numerical framework to the density of the mesh has been assessed by considering uniform grids with a progressively increasing number of points and checking the percentage variation experienced by some representative physical quantities (until such a variation falls under a given (acceptable) threshold). Such analysis has been partially supported by the use of well-known criteria for high-Ra thermal convection, these being based on the evaluation of the so-called Kolmogorov length scale. This quantity, which represents the minimum possible size of the small-scale structures present in the considered flow, is known to take different typical values in the case of thermal convection according to the relative direction of gravity and the imposed temperature gradient (see, e.g., Lappa and Gradinscak ${ }^{72}$ ). For the case of prevailing horizontal temperature gradients, with the present reference length and definition of the Rayleigh number, in particular, it can be estimated (see Paolucci ${ }^{73}$ and Farhangnia et al., ${ }^{74}$ ) as

$\eta=2 \pi\left(\frac{2 \mathrm{Pr}}{R a}\right)^{3 / 8}$

which gives $\eta=1.78 \times 10^{-2}$ for the present conditions (corresponding to approximately 60 points over a length of L, i.e. a total of 120 grid points along the vertical extension of the system).

If the prevailing temperature gradient is vertical (heating from below effect), the typical value taken by the Kolmogorov length scale (see, e.g., $\operatorname{Kerr}^{75}$ and De et al., ${ }^{76}$ ) becomes:

$\eta=1.336(R a)^{-0.32}$

For $\operatorname{Pr}=8$ and $\mathrm{Ra}=10^{8}$, this yields $\eta=3.68 \times 10^{-3}$, which would correspond to 270 points over a length of $\mathrm{L}$ (540 grid points along the vertical extension of the system).

Figure 2 shows the outcomes of the corresponding grid refinement study carried out by increasing continuously the mesh resolution from the minimum requirement indicated by eq. (13a) towards the more restrictive constraint represented by eq.(13b) (mesh convergence being expected to occur in such a range). 


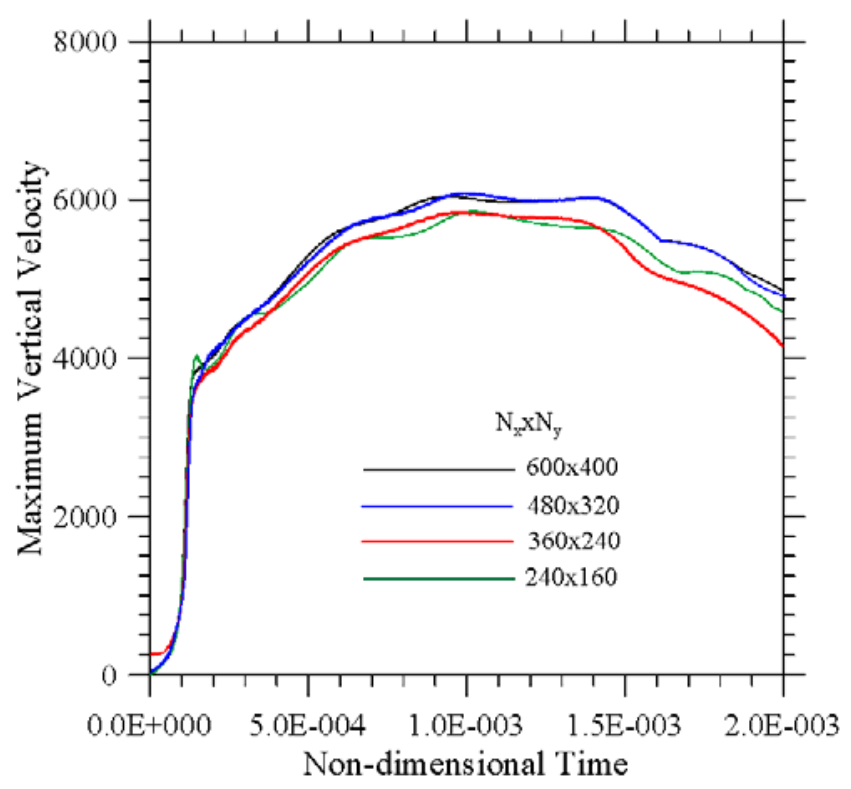

Figure 2: Maximum vertical velocity as a function of mesh resolution $\left(\operatorname{Pr}=8, \operatorname{Ra}=10^{8}\right)$.

As evident in this figure, convergence is attained when the mesh density is increased from $480 \times 320$ to $600 \times 400$ grid points (indeed, the data indicate that the related percentage variation experienced by the maximum value attained by the vertical velocity in the entire physical domain becomes smaller than $1 \%)$.

Accordingly, we have used a mesh with an intermediate number of nodes, i.e. 520 points in the $\mathrm{x}$ (vertical) direction and 360 in the transverse directions. Not surprisingly such a mesh satisfies both the requirements discussed before about the need to properly capture the smallest scale structures involved in the considered phenomena. Indeed, comparison of the chosen grid resolution (520x460) with the value of $\eta$ evaluated in the most restrictive conditions (its smallest value), namely the case in which the prevailing temperature gradient is vertical (eq. 13b), leads to the conclusion that our grid spacing is either smaller than or comparable to the Kolmogorov scale $(\Delta x / \eta$ and $\Delta y / \eta$, are approximately equal to 1 and 3/4, respectively, which is in line with the general criteria indicated by $\left.\operatorname{Ref}^{77}\right)$.

The typical (non-dimensional) time integration step used for all the simulation has been set to $10^{-8}$ (satisfying two different criteria, namely the well-known conditions for the numerical stability of the diffusive and convective equation terms appearing in both the momentum and energy equation, see, e.g., $\operatorname{Ref}^{78}$ ). No appreciable changes have been noticed on decreasing it (not shown).

Special care has been devoted to the integration of the Maxey-Riley equation as well, for which a fourth-order accurate Runge-Kutta approach has been implemented (although for the sake of concreteness it should be mentioned that, owing to the very small integration time step set for all cases $\left(\Delta \mathrm{t}=10^{-8}\right)$, the use of lower-order schemes in the integration of the Maxey-Riley equation would not have produced significant differences with respect to the application of the fourth-order accurate variant).

The CFD kernel of the present code was successfully used for numerous studies of fundamental fluid-dynamics (the reader being referred to Lappa ${ }^{20}$ for an assessment of the code performances for 
a typical problem of turbulent gravitational convection) and repeatedly validated also by comparison with other kinds of convection (of various natures, see, e.g., Refs. ${ }^{47,78}$ ).

For what concerns the assessment of the particle tracking approach against other data in the literature, relevant information can be found in $\operatorname{Ref}^{47}$ and $\operatorname{Ref}^{79}$ for the case of particles interacting with a liquid and a gas (and compressible and incompressible flows), respectively. The same subroutines were also used by $\operatorname{Ref}^{53}$. We recall here that in all these works proper coupling between the CFD and particle tracking kernels was implemented on the basis of a simple linear interpolation used to determine the fluid velocity at generic particle locations (and the related substantial derivative as required by eq. (11a)) as a function of the corresponding values taken by $\underline{V}$ on the fixed nodes of the staggered grid.

The influence of the mesh on particle dynamics for the present conditions can be gathered from Figure 3. The additional data shown there have been produced under the two-fold motivation to verify that 1) particle-dynamics converge towards a mesh-independent behaviour as the number of grid points is increased (just as the carrier velocity field does); 2) the number of considered particles is statistically relevant (which means that the observed particle "ensemble" dynamics do not depend on such a number as well). In order to address in a relevant way the second point, we have introduced some dedicated "global" parameters as

$$
Q^{+}=\frac{\sum_{u_{p a r t}>0} m_{p a r t} u_{p a r t}}{M_{\text {tot }}}, Q^{-}=\frac{\sum_{u_{p a r t}<0} m_{p a r t} u_{p a r t}}{M_{\text {tot }}}
$$

where $u_{\text {part }}$ is the vertical velocity component of each particle and $m_{\text {part }}$ its mass (the parameter $Q^{ \pm} M_{\text {tot }}$ may be regarded as a measure of the overall particle instantaneous positive or negative momentum in the system). Towards the end to determine the number of particles $\mathrm{N}_{\text {part }}$ to be exceeded in order to make the behaviour of the dispersed phase statistically relevant (i.e. not dependent on $\mathrm{N}_{\text {part }}$ ), we have scaled the number of particles effectively tracked for each simulation with the mesh as $N_{\text {part }}=N_{x} / 2 \times N_{y} / 2$. The results for a representative set of parameters $\left(\xi=0.8, \gamma=2.15 \times 10^{10}\right.$, $\mathrm{St}=2 \times 10^{-7}$ ), summarized in Fig. 3, demonstrate the reliability of the present hybrid EulerianLagrangian numerical approach. On increasing the number of points used for the Eulerian uniform structured mesh and the number of effectively tracked Lagrangian particles accordingly (in a linearly proportional way), it can be seen that beyond a certain threshold the observed dynamics became independent of both the mesh and the number of tracked particles ${ }^{79}$.

Further code validation has been achieved through comparison with the theoretically predicted sedimentation terminal velocity (eq. (10)) for cases in which the fluid is in quiescent conditions, obtaining differences between the computed solid particle velocity and the corresponding theoretical value (depending on Fr and Pr), less than 1\%. 

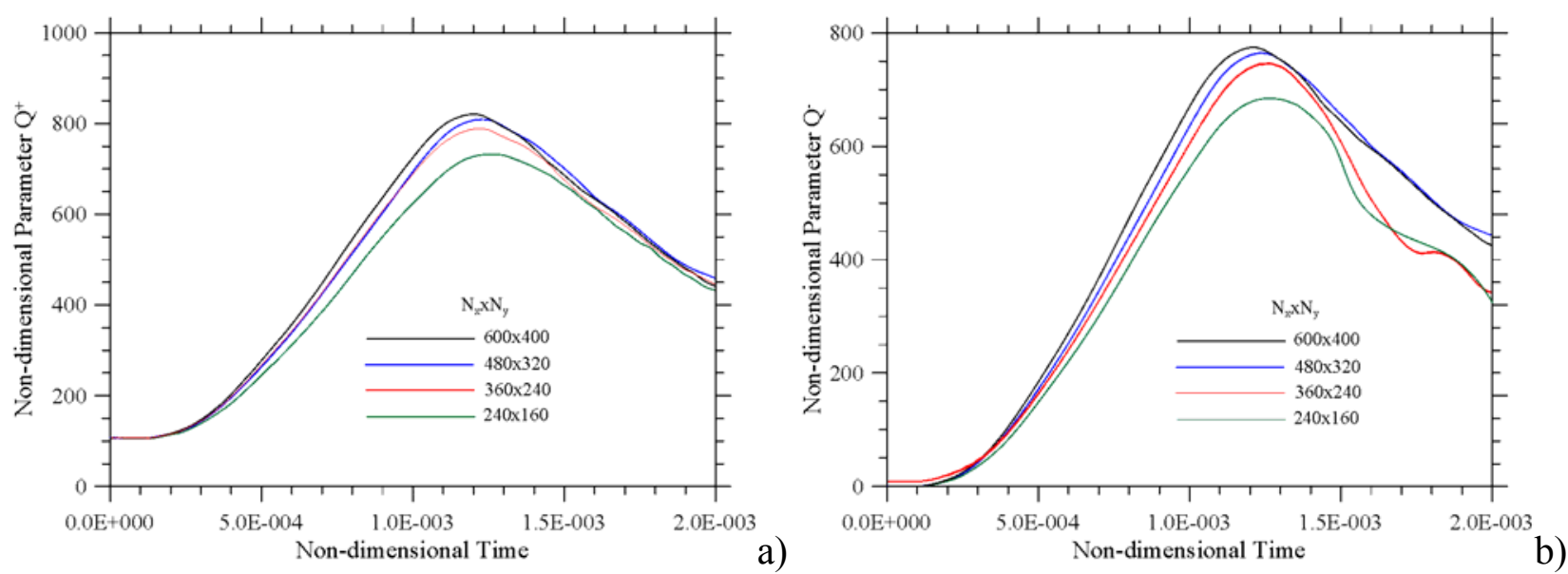

Figure 3: Parameters $Q^{+}$(A) and $Q^{-}$(b) versus time for $\xi=0.8, \gamma=2.15 \times 10^{10}\left(\mathrm{Fr}=5.45 \times 10^{-5}\right)$, $\mathrm{St}=2 \times 10^{-7}, \mathrm{Pr}=8, \mathrm{Ra}=10^{8}$ and different mesh resolutions and number of particles tracked $\left(N_{\text {part }}=N_{X} / 2\right.$ $\left.\times N_{y} / 2\right)$

\section{Results and discussion}

As discussed to a certain extent in the introduction, the main objective of the present study is to clarify the interplay between localized buoyancy convection in the form of a plume rising from the bottom of a fluid domain and particle dispersion-vs-segregation mechanisms. Periodic boundary conditions are imposed at the lateral vertical boundaries with the express intent to mimic a discrete set of spatially periodic rising plumes. This realistic configuration is typical of several natural and industrial contexts. Indeed, common examples in nature are volcanic gas eruptions (Kaminski et al., ${ }^{80}$ ) and hydrothermal vent flows in the deep ocean; they are also a typical feature of modern society's fluid waste disposal methods including gaseous emissions into the atmosphere from domestic and industrial smokestacks and thermal discharges from nuclear and fossil-fueled electricity generation plants (Lee and Asce $^{81}$ ); configurations with multiple plumes can also exist at a smaller scale in combustion chambers as a result of the presence of holes or orifices for fuel injection and dilution (Issac and Jakubowski ${ }^{82}$ ).

As anticipated in Sect.2, the shape of the particles is fixed (spherical). An important degree of freedom, however, is introduced in the problem by changing their viscous drag through a proper tuning of the related Stokes number $\mathrm{St}\left(\mathrm{O}\left(10^{-8}\right) \leq \mathrm{St} \leq \mathrm{O}\left(10^{-6}\right)\right)$, which corresponds to a change in the size of the particles. Similarly, the effects of inertia and gravity are varied by performing the numerical simulations for different values of the density ratio $\xi(0.2 \leq \xi \leq 2)$ and the Froude number $\left(3.85 \times 10^{-5} \leq F r \leq 8 \times 10^{-5}\right)$, corresponding to $10^{10} \leq \gamma \leq 4.3 \times 10^{10}$. Accordingly, a total of 108 simulations have been performed (each simulation requiring approximately one day of computational time on each CPU core).

To properly track the system evolution, a huge number of solid particles $\left(N_{\text {part }}=N_{x} / 2 \times N_{y} / 2 \cong 5 \times 10^{4}\right)$ have been initially seeded uniformly into the computational domain. By virtue of the one-way 
coupling assumption (governing the interactions between the liquid and solid phases, as explained at the end of Sect. 2.3), the considered number of particles has no effect on the fluid flow properties. As already explained to a certain extent in Sects. 2.3 and 2.4, our consideration of such a large number obeys a specific and rational (two-fold) need: 1) the system dynamics can be easily characterized in terms of patterning behaviour (namely, the recognizable topology of structures formed by aligned particles in the physical domain); 2) considering a statistically relevant number of solid particles makes related results and conclusions sufficiently independent and general; in other words, this framework lets us identify the average behaviour of particles by revealing the effective mean evolution (a similar approach was implemented by Raju and Meiburg ${ }^{32,36}$ ). We finally link the resulting statistics to the dynamics of the temporally evolving rising thermal plume, to provide useful information about the particle transport mechanisms and associated dissipative dynamics.

\subsection{The temporally evolving rising thermal plume}

Before starting to deal with particle phenomena and in order to improve clarity, in this section first we describe the temporal evolution of the considered background flow, i.e. the rising thermal plume shown in Fig. 4. As mentioned before, we concentrate on the so-called IVND regime in which plumes typically undergo shear-driven "sinuous instabilities". As such instabilities are known to be initially two-dimensional and eventually produce appreciable three-dimensional effects only when higher-order bifurcations are enabled, we limit our analysis to the first flow bifurcation (by which the initial symmetry of the plume with respect to the vertical direction is broken; the interested reader being referred to Desrayaud and Lauriat ${ }^{83}$ for some interesting information about the subsequent instabilities). Along these lines, in the following we identify and comment on some representative stages of plume evolution that we will expressly recall later as "reference fluid-dynamic states" for further analyses (to illustrate how the behaviour of particles changes in response to a variation of the parameters $\xi$, St and Fr for a given (fixed) background flow). This specific approach is instrumental in unravelling processes that are otherwise interwoven or overshadowed and difficult to discern.

At the beginning $(t=0)$, the fluid is in quiescent and thermally uniform conditions $(T=0, V=0)$. As shown in Fig. 4a, however as time passes, the liquid surrounding the heat source (represented by the short portion of non-dimensional length $\delta=\mathrm{d} / \mathrm{L}$ at temperature $\mathrm{T}=1$ ) becomes progressively warmer, thereby making the buoyancy mechanism operative. In particular, it can be seen that as the heated fluid rises, it pushes aside the material above it and is, itself, in turn deflected. The rising material produces a stalk, while the deflected fluid produces a cap on top. As the pushing and deflection mechanism is iterated, the edge of the cap may undergo additional deformation. The result is something that looks like a mushroom (Fig. 4a-c).

The "nondiffusive" nature of the regime (due to the relatively high value of the Rayleigh number considered, $\mathrm{Ra}=10^{8}$ ) can be clearly observed in both the temperature and velocity fields. The plume has a thin, sharp stem with a well-defined cap and lobes that are significantly deformed by symmetric vortex structures (Hier Majumder et al., ${ }^{21}$ ). 
When the top wall impedes further rise of the plume front, however, the typical cap shape discussed before is significantly deformed by its interaction with the horizontal boundary (Fig. 4f). Hot fluid previously moving essentially in the plume stem (the jet) along the vertical direction (from bottom to top) is now spread along this boundary towards both left and right corners of the computational domain. Due to continuity effects and the periodic boundary conditions imposed at the two sides, this fluid is finally pushed again towards the bottom along both lateral boundaries (return flow) thereby creating two anti-symmetric heat circulation loops.

When such complete loops are established (with warm fluid rising at $y=0$ and descending at $y= \pm 1 / 2$ ), vorticity formerly concentrated essentially in the plume lobes (Figs. 4a-4e), spreads over the entire domain producing quite a complex pattern. In particular, well-defined secondary vortices are created at the lower edges of the descending branches which constitute the return flow.

As anticipated, the tendency to develop (as time passes) diffused vorticity and the presence of strong gradients of velocity make this regime very susceptible to the onset of sinuous instabilities created by the destabilizing shear stresses between ascending and descending portions of fluid. As illustrated, e.g., by Desrayaud and Lauriat ${ }^{83}$, and Hernandez ${ }^{65}$ for the cases in which the environment where the plumes develop is not unbounded, but relatively limited, the finite size of the considered geometry does indeed play a role in determining unstable plume behaviour and evolution. This behaviour is generally referred to (see, e.g., the experimental work of Noto ${ }^{84}$ ) as the natural swaying motion of confined plumes.

This is very evident in Figs. 4k-4o where it can be seen that with time the plume stem develops a sinuous, snake-like appearance due to horizontal shear. This shear can cause the plume stem to become so distorted that the original plume collapses and new plumes develop from the original plume stem (Cortese and Balachandar ${ }^{85}$ ).

As anticipated, in this section we use such numerical results to provide some important clues regarding the expected nature of the interaction between the considered natural flow and the particles in advance.

More precisely, resolving this question requires separating the different contributions to the particle dynamics and estimating the relative importance of two fundamental mechanisms running in parallel: how particle settling (or flotation) under gravity is modified by the presence of the rising plume and how, in turn, the particle dispersion process by the coherent plume vortex structures (reported separately for the convenience of the reader in Fig. 5) is affected by gravity. 

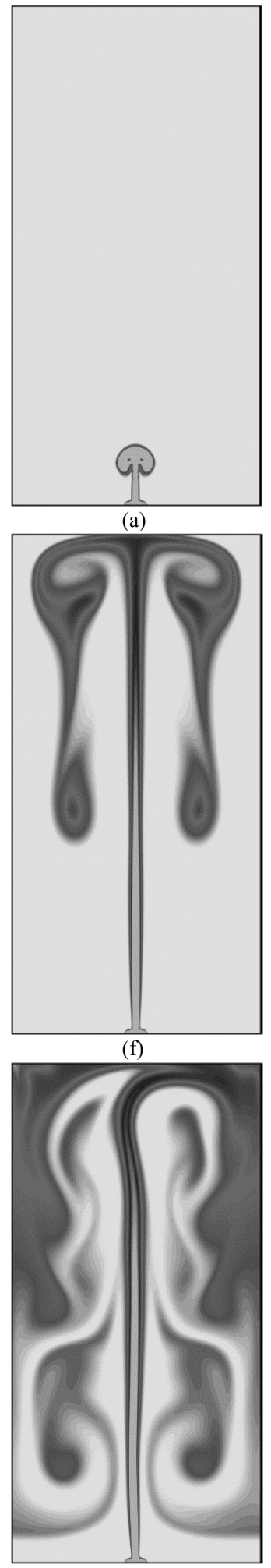

(k)

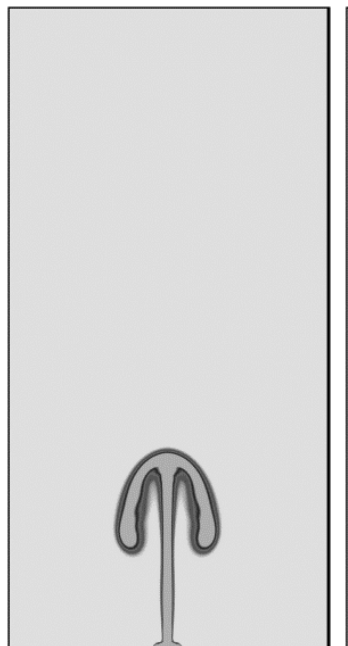

(b)

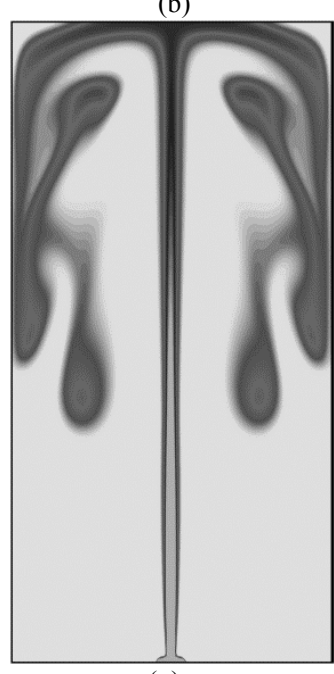

(g)

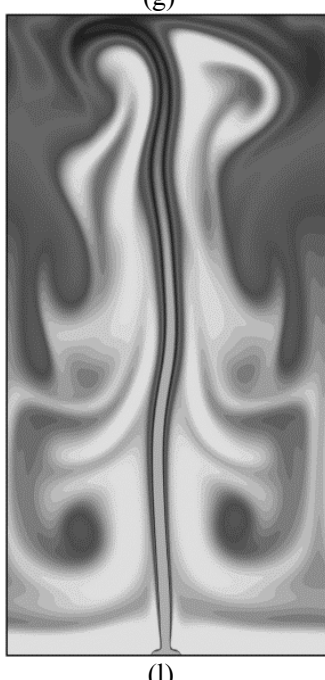

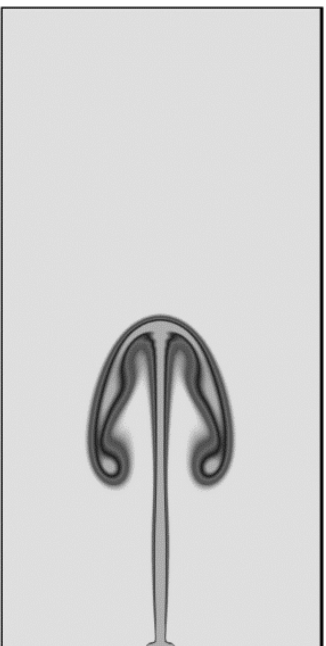

(c)

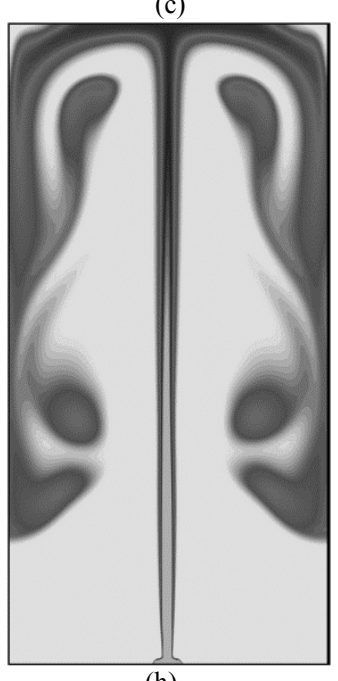

(h)

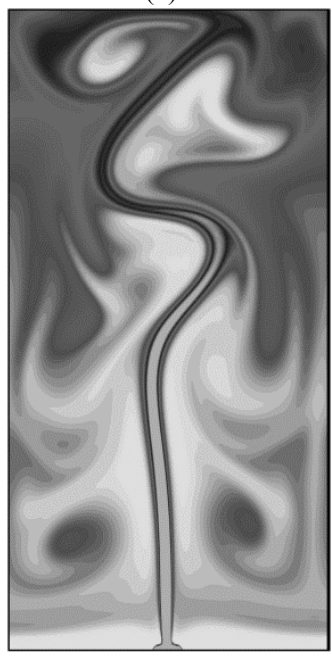

(m)
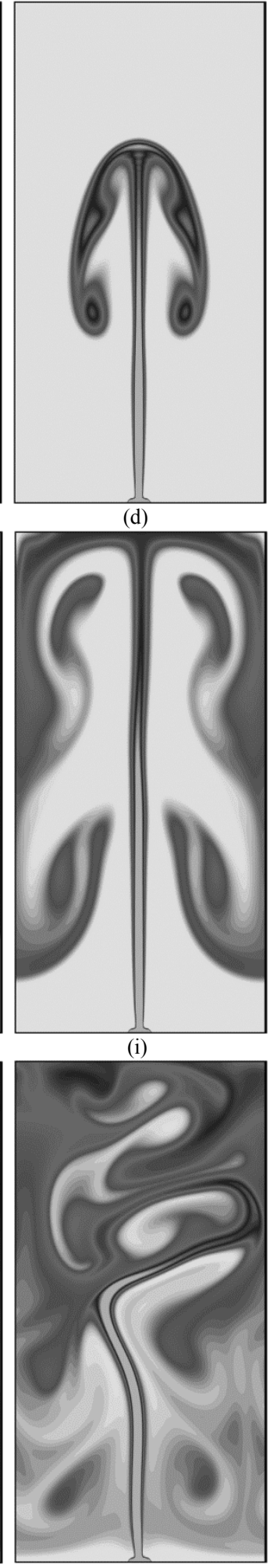

(n)
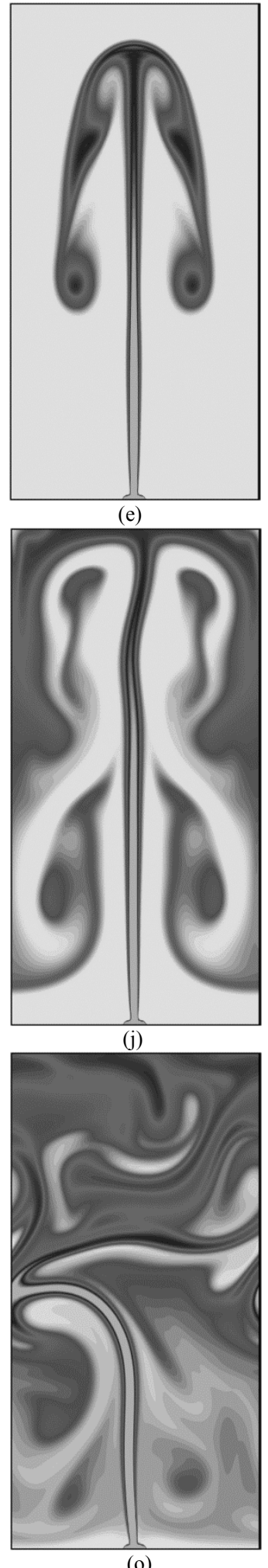

Figure 4: Snapshots of temperature distribution at different instants evenly spaced in time over the timeframe $0<\mathrm{t}<3 \times 10^{-3}\left(\operatorname{Pr}=8, \operatorname{Ra}=10^{8}\right)$. 


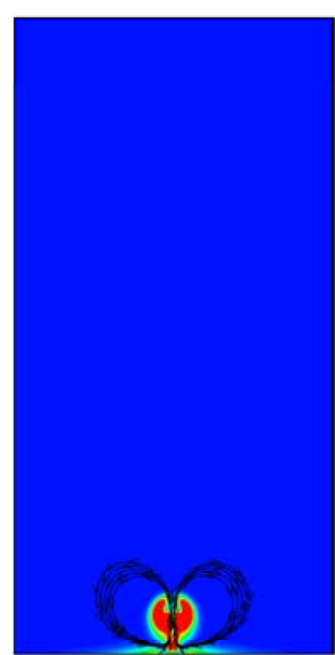

(a)
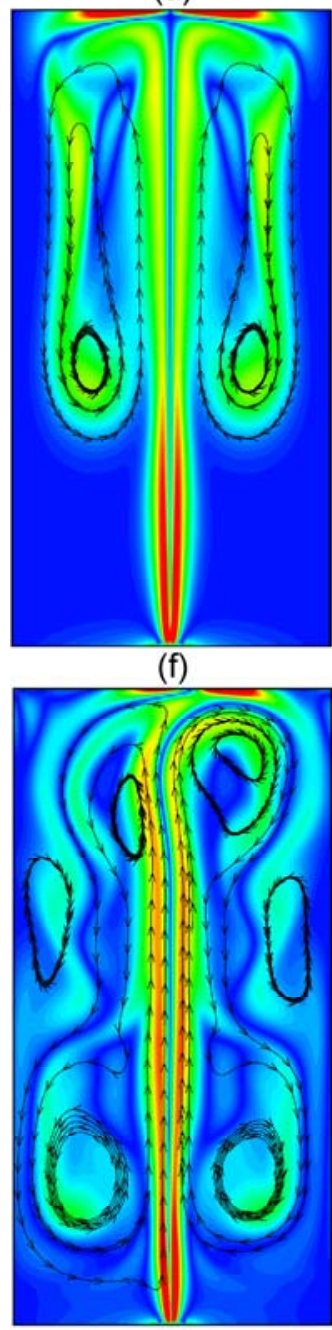

(k)

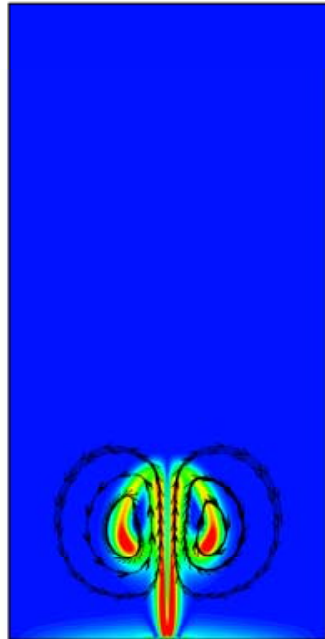

(b)

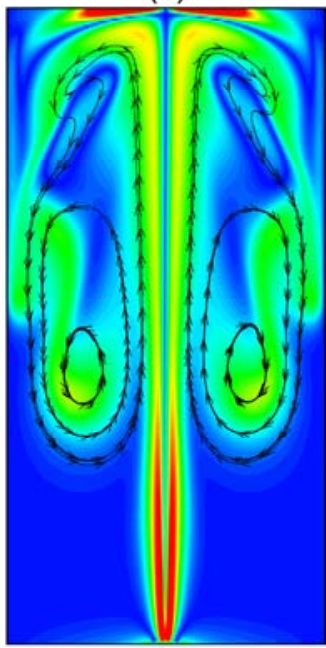

(g)

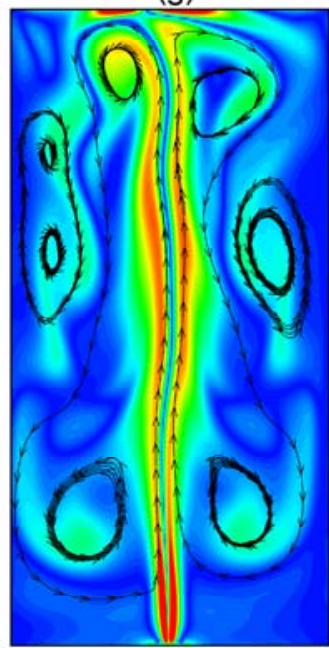

(I)

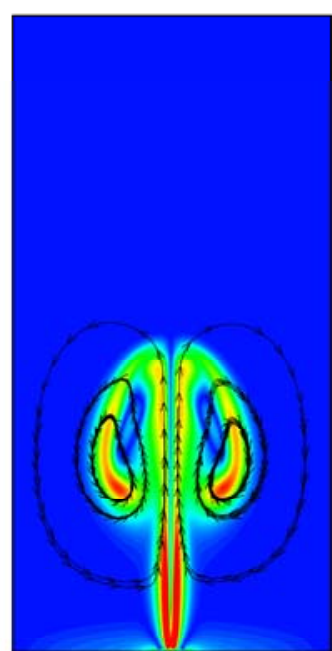

(c)

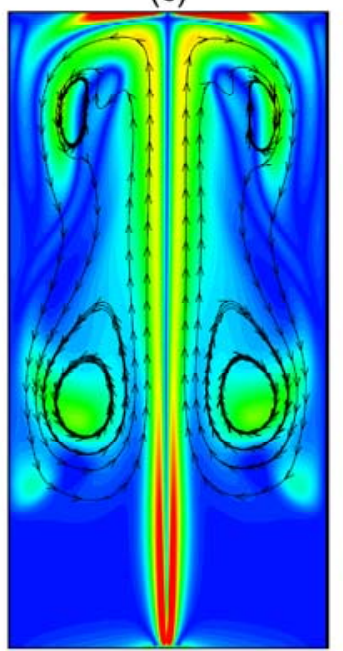

(h)

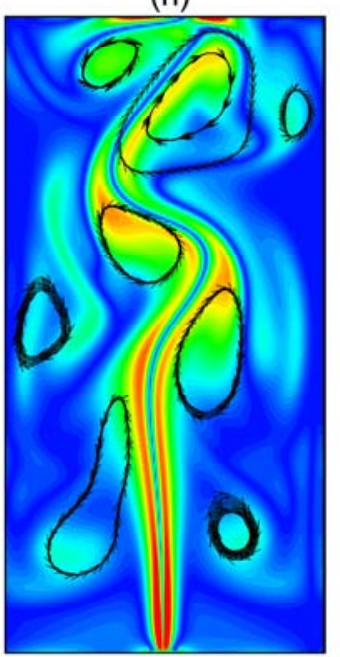

(m)

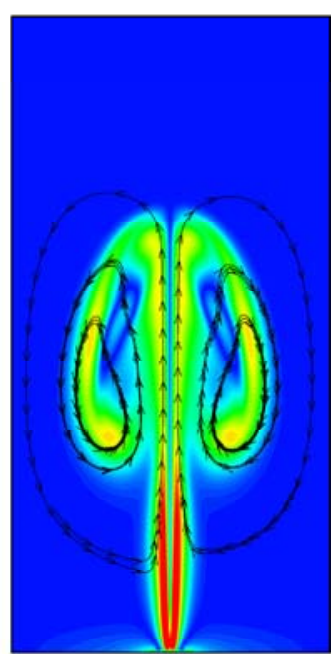

(d)
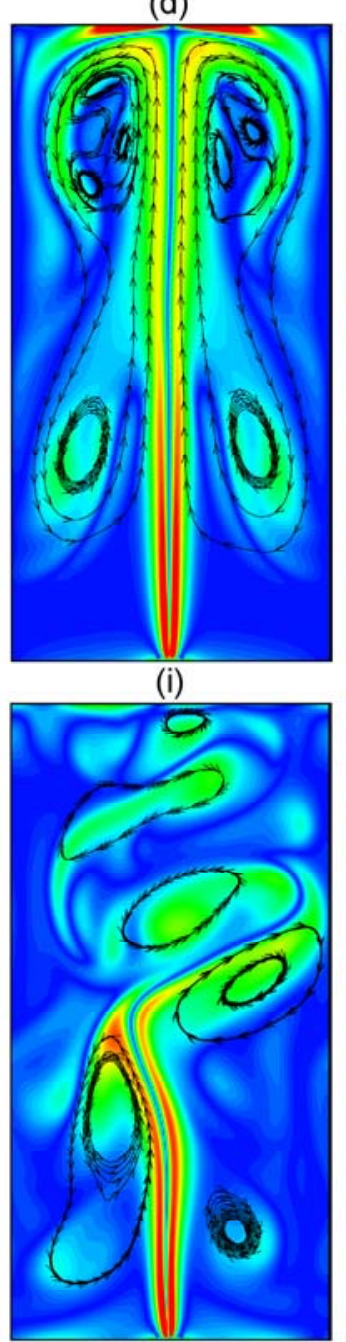

(n)

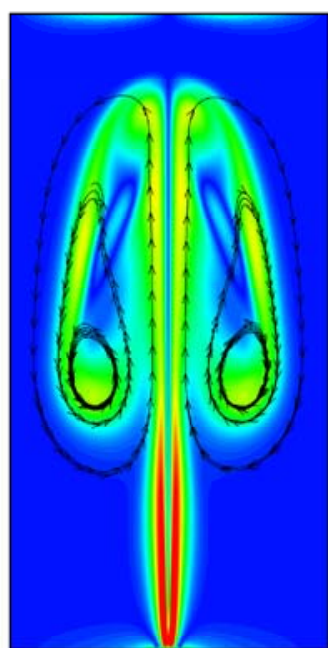

(e)

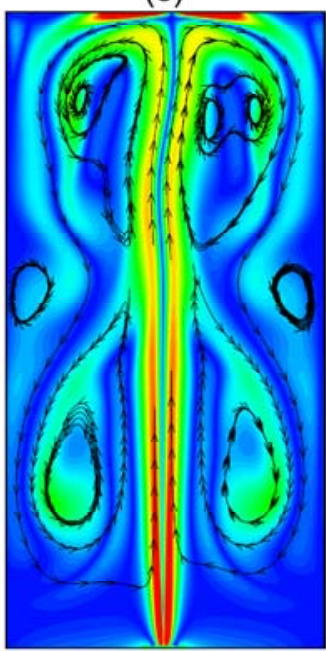

(j)

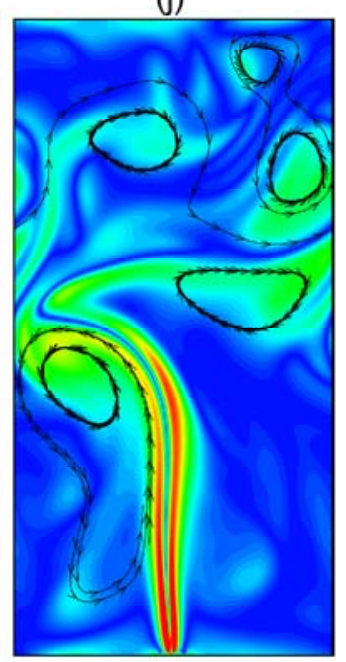

(o)

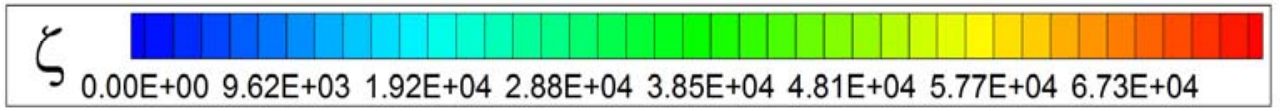

Figure 5: Snapshots of vorticity distribution (absolute value) at different instants evenly spaced in time over the timeframe $0<\mathrm{t}<3 \times 10^{-3}\left(\operatorname{Pr}=8, \mathrm{Ra}=10^{8}\right), \zeta=\partial \mathrm{v} / \partial \mathrm{x}-\partial \mathrm{u} / \partial \mathrm{y}$. 
Obviously, owing to the presence of the abovementioned strong gradients of velocity (especially between the plume stem and the surrounding stagnant fluid) and concentration of vorticity in the two lateral lobes of the plume (in the initial stages of plume development, see Figs. 5a-5e), one would expect the fluid velocity field to create quite strong gradients of particle concentration even in the absence of gravity, especially for intermediate values of St. Vice versa, one should also take into account that in a still fluid, gravity would not create concentration gradients (because it would uniformly accelerate all the particles without creating any velocity differences among them, see Raju and Meiburg ${ }^{32}$ ). With such initial arguments in mind, one may finally argue that the only way gravity can influence the emergence of inhomogeineities in the distribution of solid matter is via the interaction of the particle sedimentation (or flotation) process with the rising plume. An "a priori" classification of such effects, in turn, can be attempted as follows:

- dynamics produced by the strong rising jet established in the plume stem (and associated horizontal gradients of vertical velocity);

- effects related to the two main vortices produced by the rising plume (vorticity role);

- phenomena related to the interaction of the rising plume front with descending particles (formation of dynamical equilibrium points - related mechanisms being operative essentially in the early stages of plume development);

- effects related to shear instabilities and symmetry breaking phenomena (related mechanisms being effective in the final stages of evolution of the thermo-fluiddynamic field).

\subsection{Particles heavier than the fluid}

Comparison step by step of thermal fields and particle distribution for $\xi>1$, i.e. particles heavier that the fluid (see, e.g., Fig. 6), reveals that, for the specific ranges of Stokes and Froude numbers considered in the present analysis, heavy inertial particles can behave as "intelligent tracers" marking areas with positive buoyancy (warm fluid regions, see Fig. 4) or with significant vorticity (Fig. 5) in a very special way.

Such a statement, apparently naive, can be justified on the basis of an articulated explanation involving separate examination of the dynamics in distinct regions of the considered fluid domain. As explained to a certain extent in Sect. 3.1, using the fluid velocity, its direction and main spatial gradients as the "separation criteria", we can roughly divide the fluid domain (especially in the early stages of plume evolution) into three main areas: the central jet, the plume cap (and related lobes) and the remaining fluid (which is in more or less stagnant conditions).

In such areas particle behaviour is expected to be very different due to particle inertia (finite size and mass). The best way to make such differences evident is to concentrate on the different recognizable 
"textures" which are created by solid matter in the distinct regions of the domain according to the prevailing effect (Fig. 7).
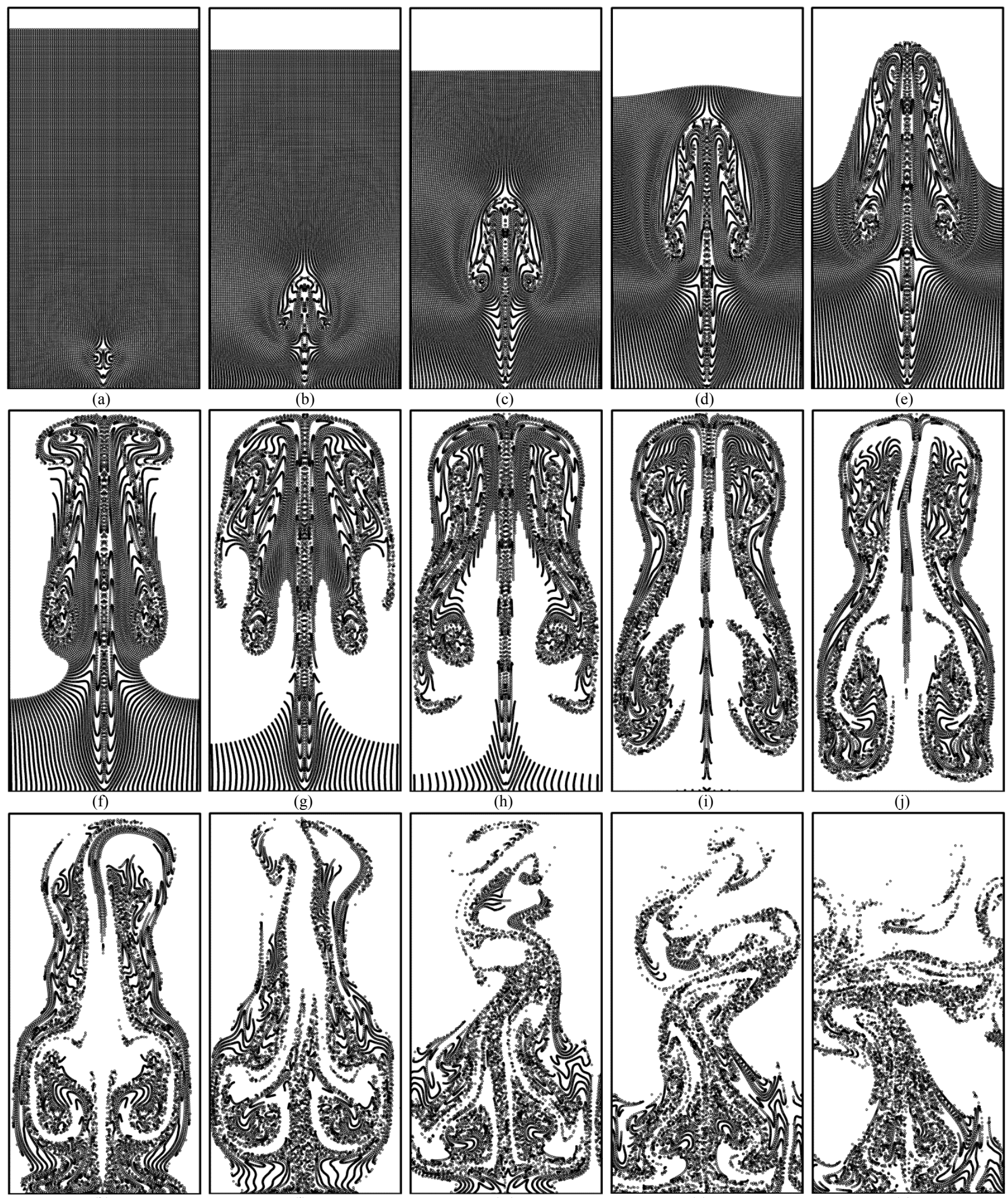

(k)

(1)

(n)

(o)

Figure 6: Particle distribution shown in different snapshots evenly distributed over the timeframe $0<\mathrm{t}<3 \times 10^{-3}\left(\xi=2, \mathrm{St}=2 \times 10^{-7}, \gamma=2.15 \times 10^{10}, \mathrm{Fr}=5.45 \times 10^{-5}\right)$. 


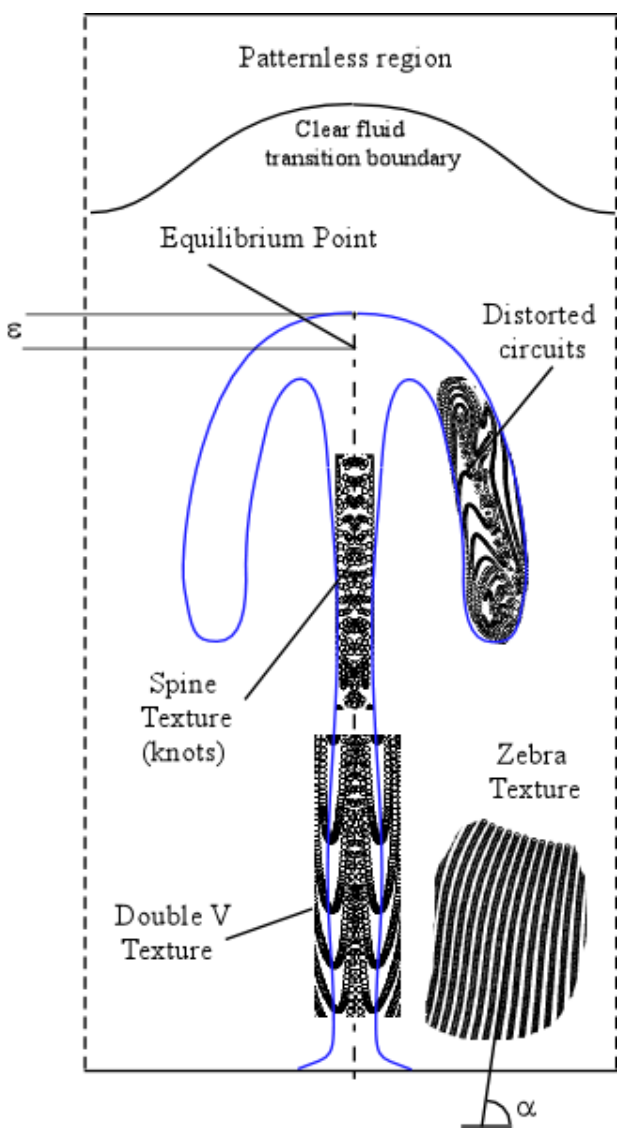

Figure 7: Regions of with different patterning behaviour (sketch) - The particles "decorate" the plume and the surrounding fluid with different recognizable textures.

Indeed, as shown by the numerical simulations, in the plume stem (the jet) where the vertical fluid velocity attains its maximum value and its gradients are particularly strong both in the axial and horizontal directions (due to vertical buoyancy and interaction with the external stagnant fluid, respectively) particles tend to form strange knot-like aggregates which give the illusion of a vertical "spine" embedded in the plume.

Similarly, in the two plume lobes, where the prevailing effects are represented by horizontal gradients of vertical fluid velocity and vertical gradients of horizontal velocity (essentially, the two components of vorticity visible in Fig. 5), particle inertia leads to the irregular but recognizable succession of particle-dense lines and well-defined near-empty spaces.

Such particle-dense lines have the shape of distorted closed circuits embedded in the two lobes of the plume cap. A thorough understanding of this phenomenon requires considering that the topology of such closed lines formed by dispersed matter depends essentially on the presence of concentrated vorticity in the plume lobes that tends to propel particles along curved (closed in many circumstances) trajectories, thereby trapping them in closed "loops" or circuits (similar findings are due for instance to Akbar et al., ${ }^{44}$ ). The rhythmic alternation of concentric particle-depleted regions and particle circuits, in turn, may be regarded essentially a consequence of particle inertia (as witnessed by the fact that for a fixed particle-to-liquid density ratio $\xi$, the spacing behaves as a function the considered value of the Stokes number, Fig. 8). 


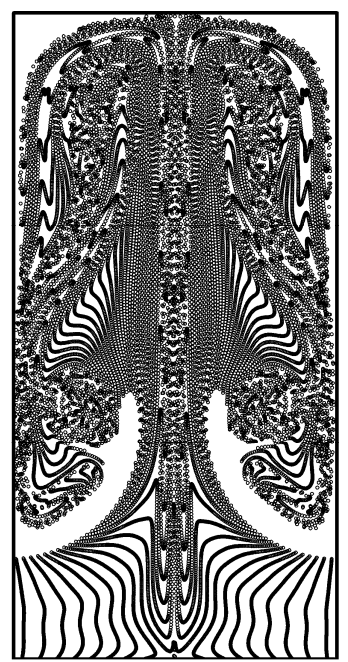

(a)

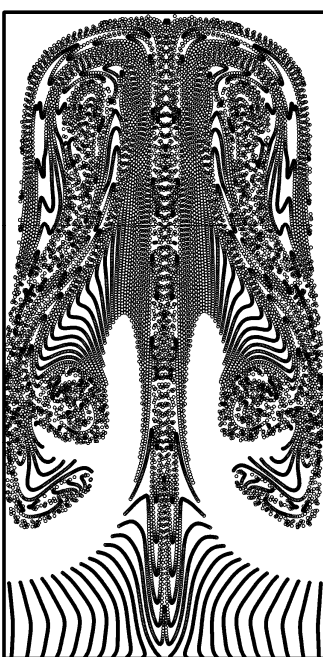

(b)

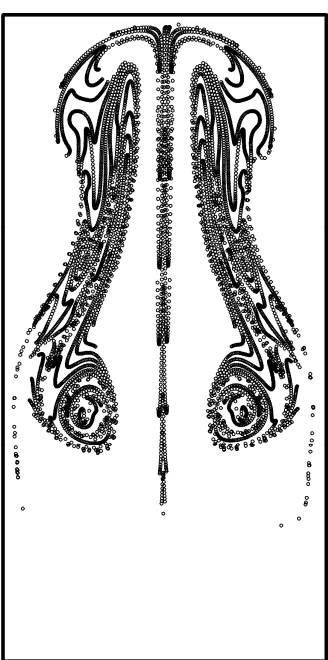

(c)

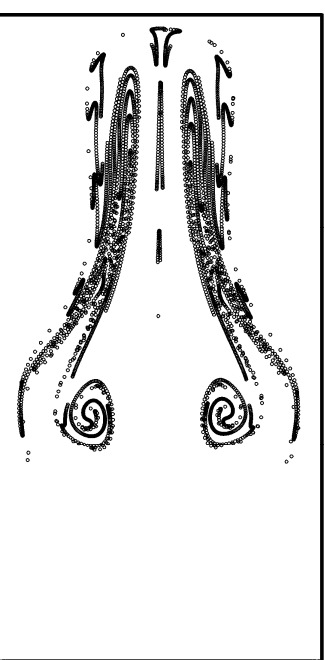

(d)

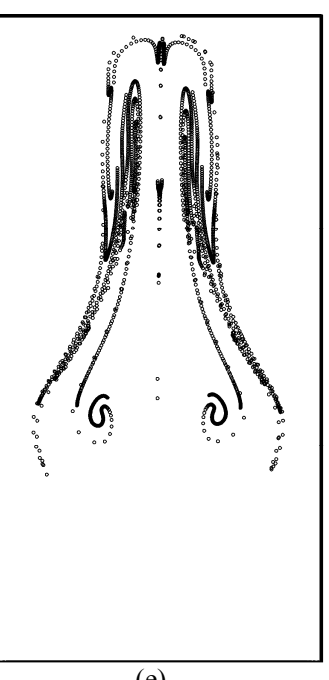

(e)

Figure 8: Snapshot of particle distribution at $\mathrm{t}=4 \times 10^{-4}\left(\xi=2, \gamma=2.15 \times 10^{10}, \mathrm{Fr}=5.45 \times 10^{-5}\right)$ for different values of the Stokes number: a) $\mathrm{St}=5 \times 10^{-8}$; b) $\mathrm{St}=1 \times 10^{-7}$; c) $\mathrm{St}=4 \times 10^{-7}$; d) $\mathrm{St}=6 \times 10^{-7}$; e) $\mathrm{St}=8 \times 10^{-7}$.

Finally, the distortion of the loops must be ascribed to the specific nature of the considered flow (which is intrinsically unsteady) and to gravity. The particle-trapping vortices are neither stationary nor fixed in space (as time passes they move towards the top). When as time progresses, the particledense lines undergo the convective transport towards the top due to the large-scale buoyancy flow, such loops are stretched and deformed. Superimposed on this are the typical effects experienced by non-neutrally buoyant, finite-sized particles: although in the region affected by plume vortices the dominant effect in producing particle motion is represented by convective transport, we must still keep in mind that particle are not passive tracers and as such they are subjected to the centrifugal force, which tends to display them outwards and the gravity force, which tends to pull them down (Eaton and Fessler ${ }^{31}$; Raju and Meiburg ${ }^{32}$; Ravichandran et al., ${ }^{40}$ ).

In general, by having an influence on the "efficiency" by which the convective flow is able to transport them (or, in other words, on the ability with which particles are able to "follow" this unsteady flow), both the mass and size of particles (namely, the density ratio $\xi$ and the Stokes number in the nondimensional space of parameters) will definitely have an important role also in the "folding process" of the particle-dense lines (discussed before), which finally determines the extension and intricate shape of the two closed circuits discussed above.

The remaining fluid (outside plume jet and cap) can be further partitioned into two main regions: a patternless state lying on the top of the plume cap and a zebra-like pattern visible in the region limited from below by the bottom wall and from above by the plume lobes (see again Fig. 7).

Despite their spatial separation, both behaviours are the expression of a single root cause, i.e. the significant lack in these areas of vertical (positive) fluid velocity and/or vorticity induced by thermal effects. 
With no significant effects opposing to sedimentation, the region lying on the top of the plume cap is progressively depleted of particles. Consequently, a sharp boundary becomes evident between a particle-filled area and a clear fluid region, which extends downward as time increases.

A similar concept applies to the zebra-like texture visible in the lower half of the domain. There, particles undergo almost unperturbed sedimentation as witnessed by the nearly vertical orientation of the lines of the particle concentration field. The small departure of such lines from the ideal purely vertical direction that would be attained in a motion-less fluid must be attributed to the residual horizontal fluid velocity component (induced in this area by the main vortices originating from the plume lobes) that tends to transport particles from the domain sides towards the central jet.

This mechanism is sensitive to particle inertia as well (as demonstrated by the relationship between the inclination angle of such lines and the Stokes number, Figs. 8a and 8b).

The transition between the almost stagnant fluid outside the jet and high-speed fluid in the jet core determines another characteristic of the pattern in this region. Indeed, the counteracting action exerted on particles by the strong buoyancy fluid velocity in the jet core and the sedimentation process just outside is responsible for the formation of recognizable $\mathrm{V}$-shaped profiles in the particle concentration on both sides (lefty and right) of the jet core. The extension of such profiles is a function of the particle inertia ( $\xi$ and $\mathrm{St}$ ) as well.

Another interesting feature of the particle concentration field originating from the interaction of thermal buoyancy effects with the gravitational settling of solid matter is related to the accumulation of particles in a specific point located inside the plume cap.

This phenomenon is illustrated in Fig. 9, where the reader may appreciate the deceleration experienced by the sedimenting particles while approaching the plume cap from the top and, at the same time, their progressive clustering in a region of very limited extent (highlighted by the horizontal arrow).

This accumulation point, which should not be confused neither with a static locus (i.e. a point fixed in space and time), nor with a stagnation point (i.e. a point where fluid velocity is zero) should rather be regarded as a condition of dynamic equilibrium attained by particles. Using dynamic arguments in place of definitions, we may also characterize this point by observing that a particle leaving this point due to random perturbations acting along the vertical direction would be subjected to an opposing restoring force pushing it back to the equilibrium point. Particles clustered in this point, however, are still sensitive to perturbations acting along the transverse (horizontal direction), which as shown in Fig. 9 can force particle to leave the equilibrium position by flowing along the right or left part of the plume cap front. 

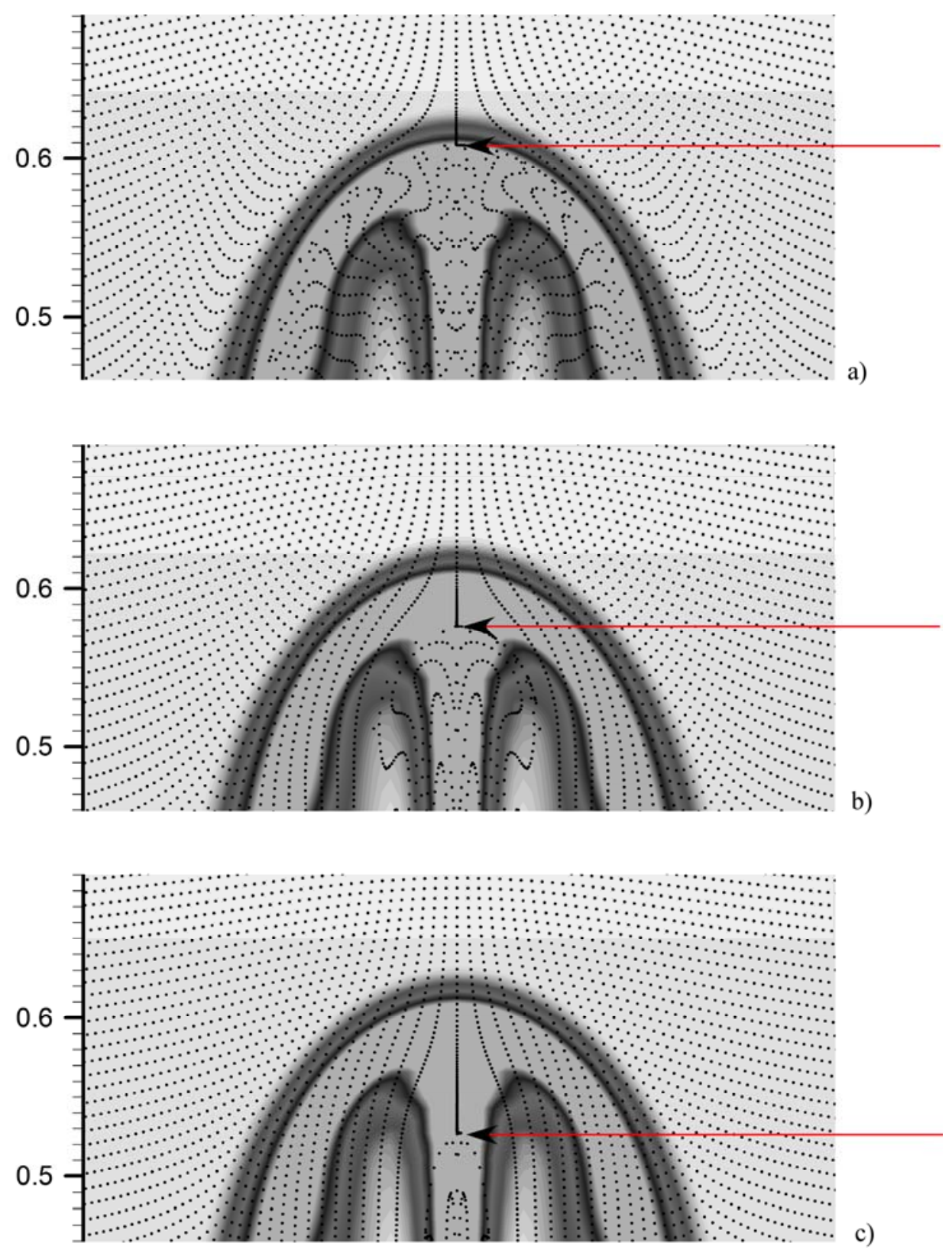

Figure 9: Dynamic equilibrium point in the early stages of plume development $\left(t=4 \times 10^{-4}\right)$ for $\xi=2.0$ and $\mathrm{Fr}=5.45 \times 10^{-5}\left(\gamma=2.15 \times 10^{10}\right)$ as a function of the Stokes number: a) $\mathrm{St}=5 \times 10^{-8}$; b) $\mathrm{St}=4 \times 10^{-7}$; c) $\mathrm{St}=8 \times 10^{-7}$.

All the above descriptions apply to the early stages of plume development.

When the plume cap hits the top boundary, thereby giving rise to a significant return (downward) flow, a clear change can be notified in the scenario.

While particles in other portions of the fluid are removed from the domain due to the endless sedimentation process, the entire plume body is still populated by dispersed particles, as made evident by particle lines that further stretch and deform in the plume interior as time passes, leading to very intricate patterns.

Characteristic behaviours are shown in Figs. 6f-6j. 
As an example, Fig. 6h shows two rounded patches "hanging" from the main particle region towards the clear fluid below. Such patches reflect another important fluid-dynamic effect, i.e. the existence of secondary vortex cores located on the edges of plume lobes (see Sect. 3.1). As time increases, these patches migrate towards the bottom with the return flow (Fig. 5).

Fig. $6 \mathrm{~g}$ also reveals that the shape of the boundary separating the particle region from the external particle-free fluid develops some cusp points, which later evolve into lateral extended branches.

Such branches, featured by the collapse of particles into thin (nearly one-dimensional) areas (in embryonic conditions in Fig. 6g), are the manifestation in the particle concentration field of the aforementioned return flow originating from the impact of the plume cap with the top wall.

As time passes, such return flows reach the bottom wall thereby producing by continuity (Figs. $6 \mathrm{~h}-6 \mathrm{j}$ ) two intense vortical structures embedded in the overall plume circulation system. Such vortices are responsible for folding and reversal in the direction of the two aforementioned descending thin particle branches. In turn, this effect produces well-defined spiralling filaments.

As time grows further, the upper half of the particle-filled plume body undergoes shrinkage in size, whereas the lower half enlarges due to the presence of the two stable vortices just discussed above. As a result, an amphora-like shape is created (the distribution of particles results in a shape that bulges out below $x=1$ and necks in above it, Fig. $6 \mathrm{j}$ and $6 \mathrm{k}$ ).

At later times, while the total number of suspended particles continues to decrease due to the solidmatter removal action exerted by gravity, a new fluid-dynamic effect comes to play a role in the dynamics, i.e. the plume instability mechanism outlined at the end of Sect. 3.1.

The outcomes of the ensuing symmetry-breaking and plume-rupture phenomena can be clearly seen in the particle concentration field (Fig. 6). The overall set of particles, which remained a cohesive entity for a long time, maintaining a sharp boundary between its interior and the clear (particle-free) fluid outside, now displays a non-compact behaviour with distinct groups of particle leaving the main particle-laden area and behaving apparently as independent "blobs" or "satellites" ejected from the main particle distribution (Figs. 6m-6o).

\subsection{Particles lighter than the fluid}

After discussing the general characteristics of the fluid-solid system in the case of heavy particles, we now turn to describing the companion dynamics for $\xi<1$.

As the reader will immediately realize by inspection of the examples shown in Figs. 10a-o for $\xi=0.2$, the change in sign of the quantity $\xi-1$ ( $>0$ for heavy particle, $<0$ for light particles) leads to dramatic modifications in the patterning behaviour and general dynamics displayed by solid matter. Along these lines, comparison step by step of Figs. 4 and 10 is instrumental in revealing the fundamental differences with respect to Figs. $6(\xi>1)$.

It needs no demonstration that because in this case particle are lighter that the surrounding fluid, they will tend to rise in any case. This tendency can immediately be seen in the evolution of the recognizable front separating the particle-rich region from the clear fluid below. 
As time progresses, this well-defined transition boundary moves upward. On such a general trend, however, are superimposed the specific effects relating to the particle-plume interactions. Such effects lead to interesting (apparently counterintuitive) consequences.

Unlike cases with $\xi>1$, where in the initial stage of evolution the plume body (jet and cap) could be essentially recognized (within the overall particle concentration field) by virtue of the specific pattern of dispersed solid matter produced by phenomena of inertial nature, now $(\xi<1)$ gravity plays (although indirectly) a more fundamental role. The entire plume is now made evident by the complete absence of particles (a patternless region).

By removing particles from the bottom of the container, gravity prevents solid mass from entering the plume jet. Only clear fluid is available close to the bottom and, accordingly, plume grows at the expenses of particle-free fluid available in proximity to its basis.

As time passes, notably, a non-compact jagged (but still ordered) distribution of particles tends to be created in the lower part of the domain (Fig. 10c-10h). This pattern, made recognizable by a set of inclined particle-lines (hereafter simply referred to as "rays" due to their diverging appearance), is produced by the weak action that the horizontal component of fluid velocity (induced by plume vortices as already explained in Sect. 3.2) exerts (in conjunction with inertial forces) on rising particles.

Most interestingly, particles aligned along the "rays" are periodically entrained by the jet. As a result, these particle lines are distorted and give rise to typical inverted-V particle profiles visible in the jet core (Figs. 10g-10i). These sets of V-shaped particle agglomerates are transported upwards by the rising jet fluid until they populate the upper part of the plume and are finally absorbed in the main particle concentration field (Fig. 10j). This process continues until all the rays are consumed and further entrainment of particles coming from this particular region is no longer possible (Fig. 10k). In line with these observations, for a further increase in time, the plume displays a completely particlefree behaviour (giving the illusion of a clear "river" flowing through a "land", Figs. 101-10o).

As opposed to cases with $\xi>1$, where such effects were counteracting, here gravity and buoyancy flow act in the same direction forcing particles to move upward. Remarkably, this prevents the solidliquid system from forming dynamic equilibrium points such as those discussed in Sect. 3.2. Rather, a dynamically evolving particle-region/clear-liquid transition boundary is created in front of the rising plume cap (Fig. 11). 

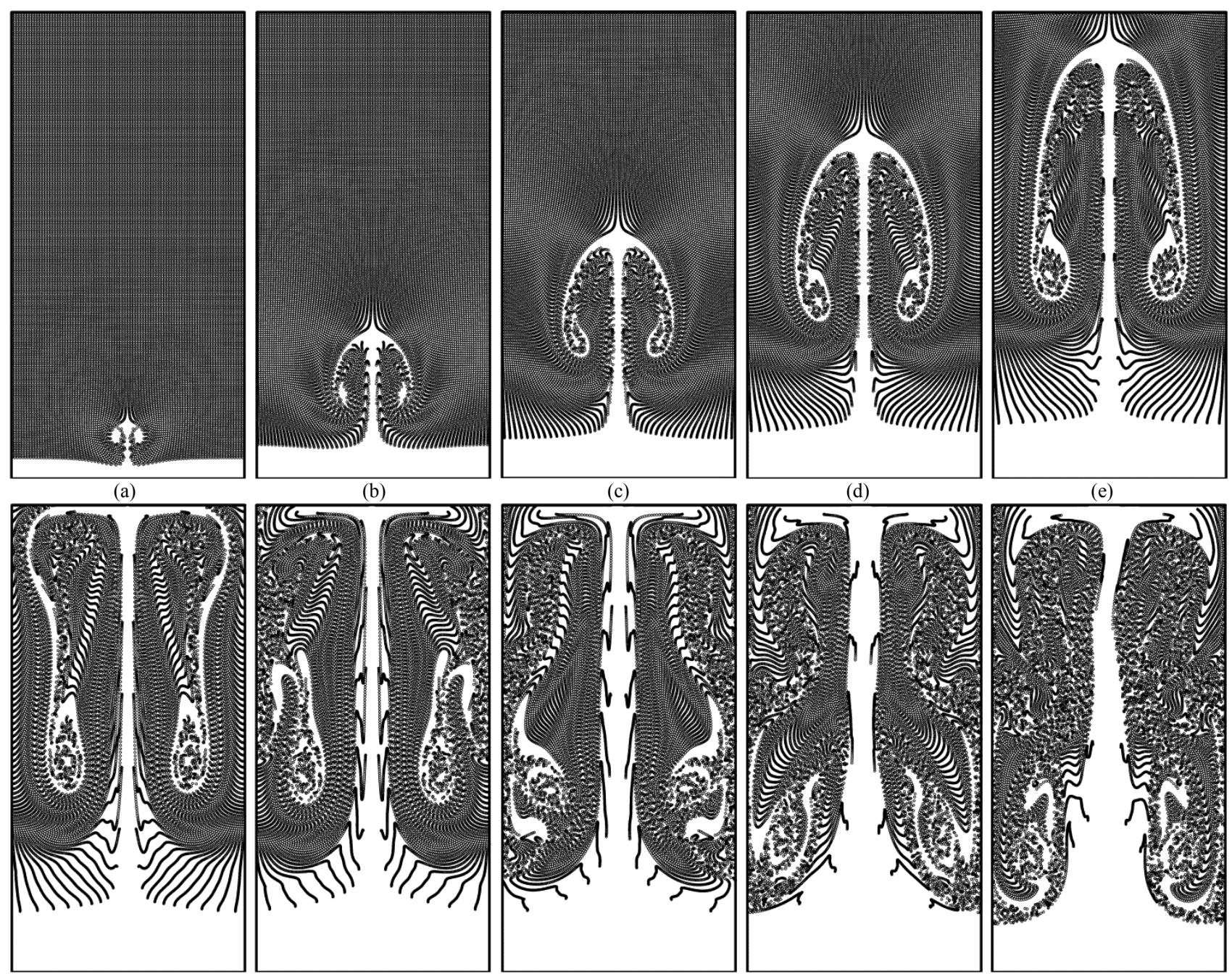

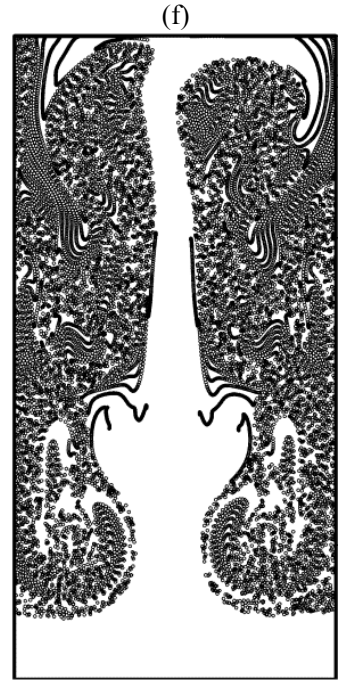

$(\mathrm{k})$

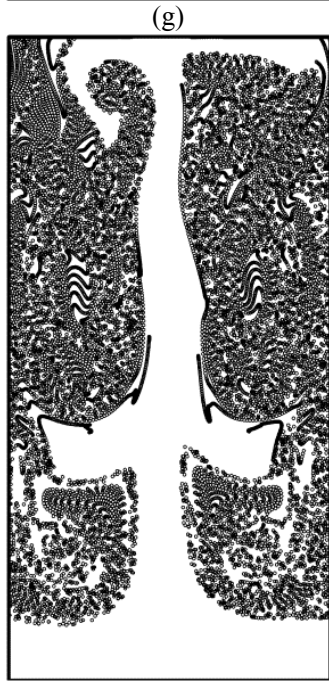

(1)

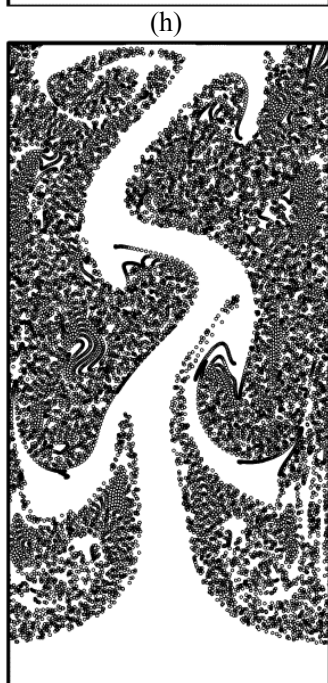

(m)

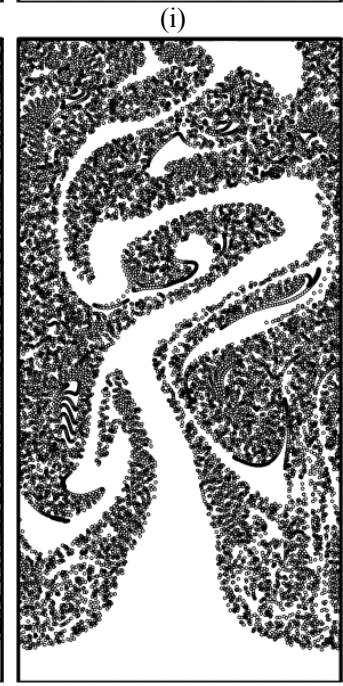

(n)

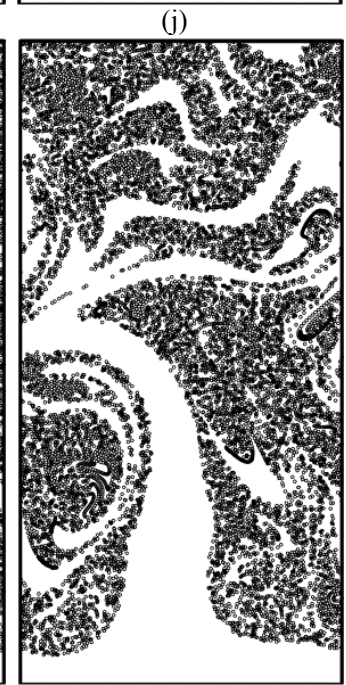

(o)

Figure 10: Particle distribution shown in different snapshots evenly distributed over the timeframe $0<\mathrm{t}<3 \times 10^{-3}\left(\xi=2 \times 10^{-1}, \mathrm{St}=2 \times 10^{-7}, \gamma=2.15 \times 10^{10}, \mathrm{Fr}=5.45 \times 10^{-5}\right)$. 


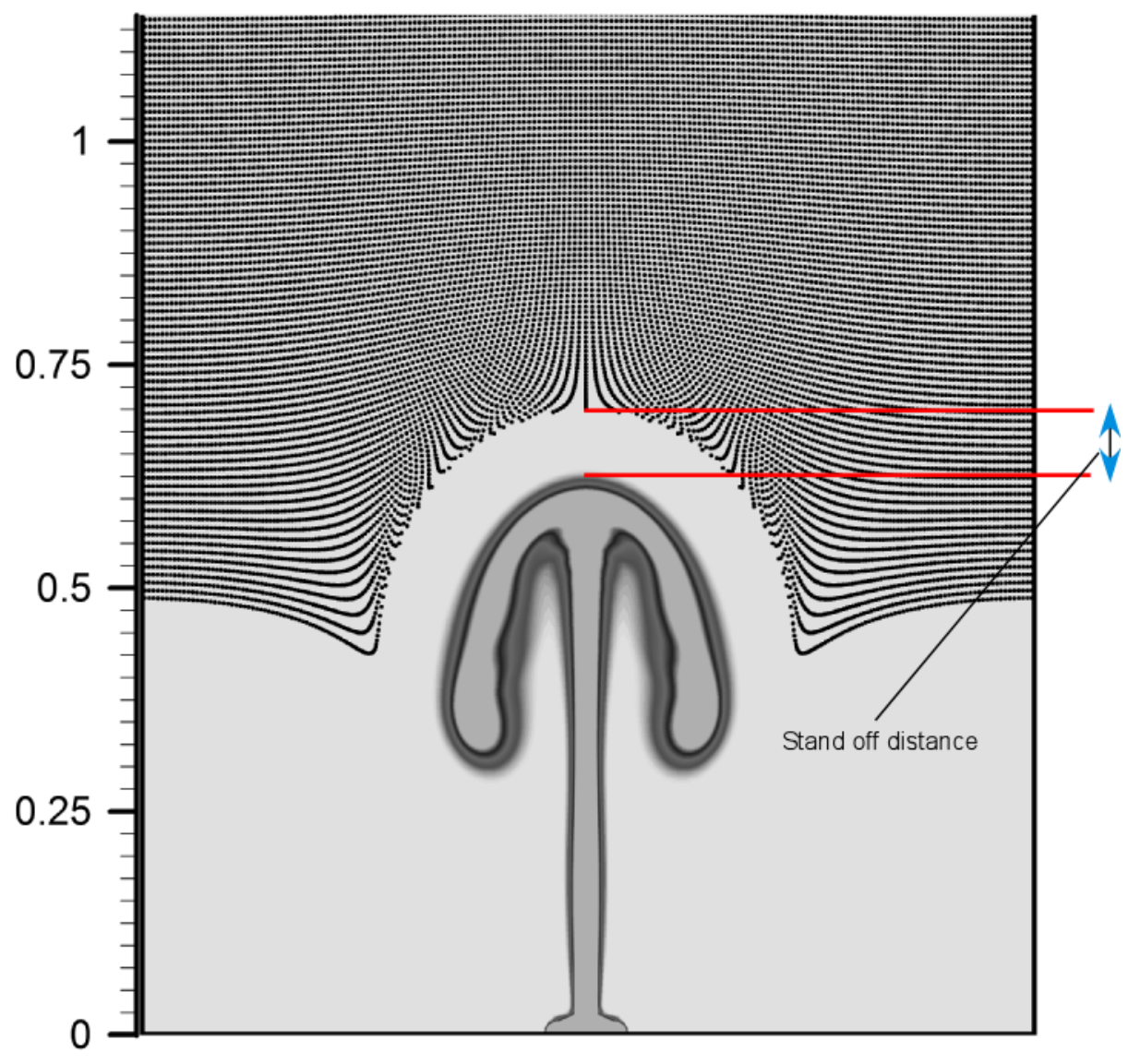

Figure 11: Snapshot of particle distribution and underlying temperature field at $\mathrm{t}=4 \times 10^{-4}$ for $\xi=0.5$, $\gamma=2.15 \times 10^{10}\left(\mathrm{Fr}=5.45 \times 10^{-5}\right)$ and $\mathrm{St}=1 \times 10^{-6}$.

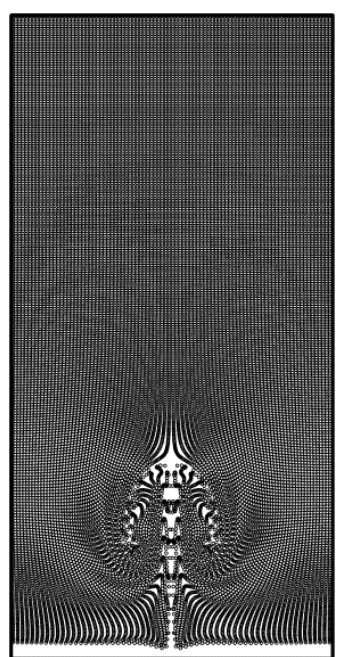

(a)

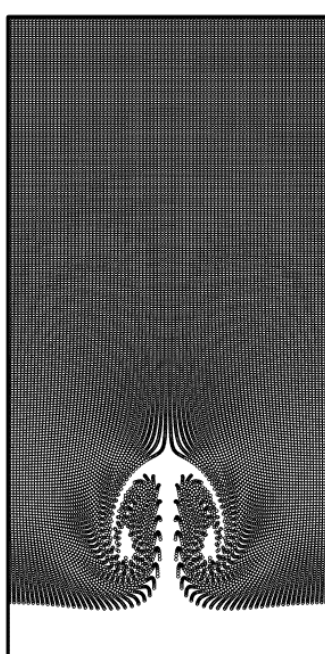

(b)

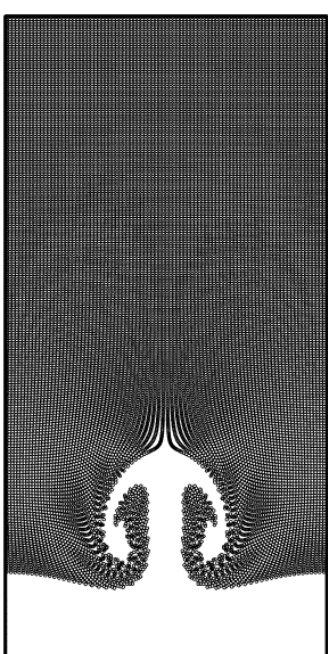

(c)

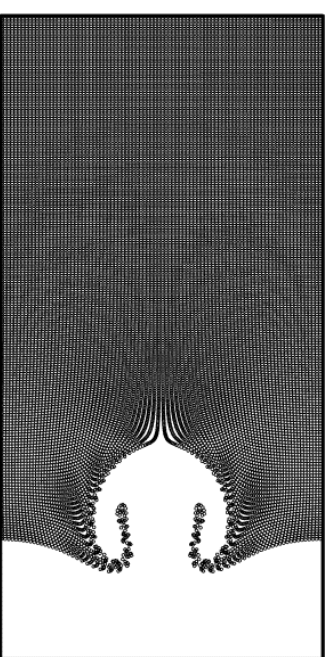

(d)

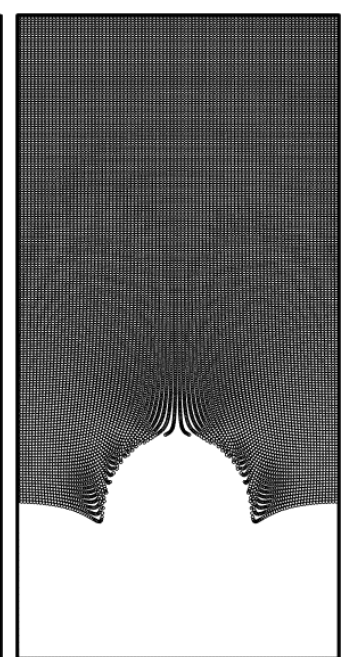

(e)

Figure 12: Snapshot of particle distribution at $\mathrm{t}=4 \times 10^{-4}\left(\xi=0.5, \gamma=2.15 \times 10^{10}, \mathrm{Fr}=5.45 \times 10^{-5}\right)$ for different values of the Stokes number: a) $\mathrm{St}=1 \times 10^{-7}$; b) $\mathrm{St}=4 \times 10^{-7}$; c) $\mathrm{St}=6 \times 10^{-7}$; d) $\mathrm{St}=8 \times 10^{-7}$; e) $\mathrm{St}=1 \times 10^{-6}$. 
The role particle inertia does still play in such dynamics can be elucidated by showing the particle distribution as a function of the Stokes number (Fig. 12). A remarkable effect can be seen there in the morphology of the particle-free space produced by the plume interaction with the particle distribution (the shape of the boundary separating the particle-rich region from the clear fluid). The shape at a given instant ranges from arrow-headed one to elliptic or almost perfectly circular.

\subsection{Effect of density ratio and Froude number}

Some final room should also be devoted to discuss the interesting information that can be gathered via a parametric analysis of the system behaviour at fixed values of the Stokes number and as a function of the density ratio and the Froude number (Fig. 13).

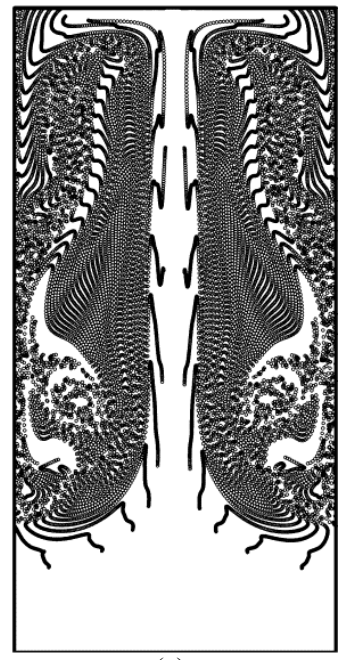

(a)

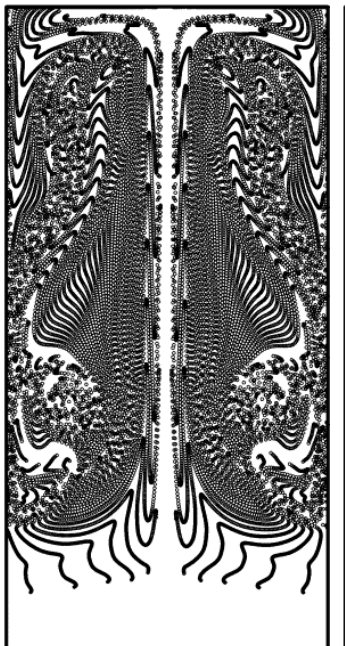

(b)

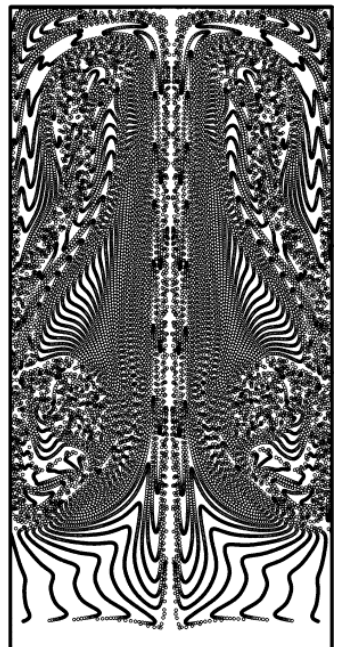

(c)

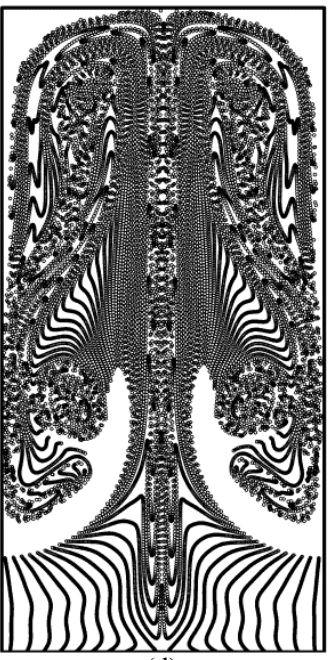

(d)

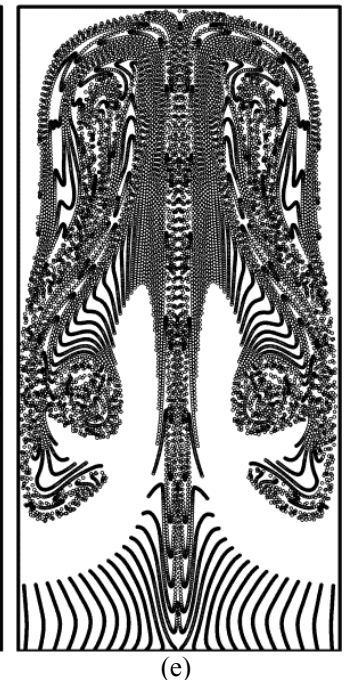

(e)

Figure 13: Snapshot of particle distribution at $\mathrm{t}=1.6 \times 10^{-3}\left(\mathrm{St}=2 \times 10^{-7}, \gamma=2.15 \times 10^{10}, \mathrm{Fr}=5.45 \times 10^{-5}\right)$ for different values of the density ratio $\xi$ : a) $\xi=0.2$; b) $\xi=0.5$; c) $\xi=0.8$; d) $\xi=1.3$; e) $\xi=1.6$.

Interestingly, Fig. 13 gives further evidence to some of the arguments already elaborated in Sect. 3.2 about the capacity of plume vortices to temporarily "trap" particles in distorted (elongated vertically) closed circuits over a wide range of $\xi$, with the formation of $\mathrm{V}$-shaped particle profiles in the central region separating such vortices (V apex down for $\xi>1$, up for $\xi<1$, respectively). Although the dynamics of particles become increasingly different for $\xi>1$ and $\xi<1$ as time increases, a limited timeframe can be identified in which they tend to be trapped in the two vortices giving rise to qualitatively similar patterning behaviours in the region enclosed in the corresponding two plume lobes.

Additional, specific insights into the general dynamics can be sought from consideration of the parameters $Q^{ \pm}$as a function of time (Fig. 14).

As expected, for a fixed St, the relative importance of $Q^{+}$and $Q^{-}$changes according to the value of $\xi$, with $Q^{+}$lying above $Q^{-}$for $\xi<1$ and vice versa for $\xi>1$. In these plots, the non-monotone behaviour of $Q^{ \pm}$(initially increasing, then decreasing with time) is intimately related to the transient dynamics of the rising plume that, by virtue of its own fluid velocity (especially in the jet and two side 
vortices), tends to interfere with the otherwise uniform and homogeneous particle sedimentation (or flotation) process, as widely discussed in the preceding sections.
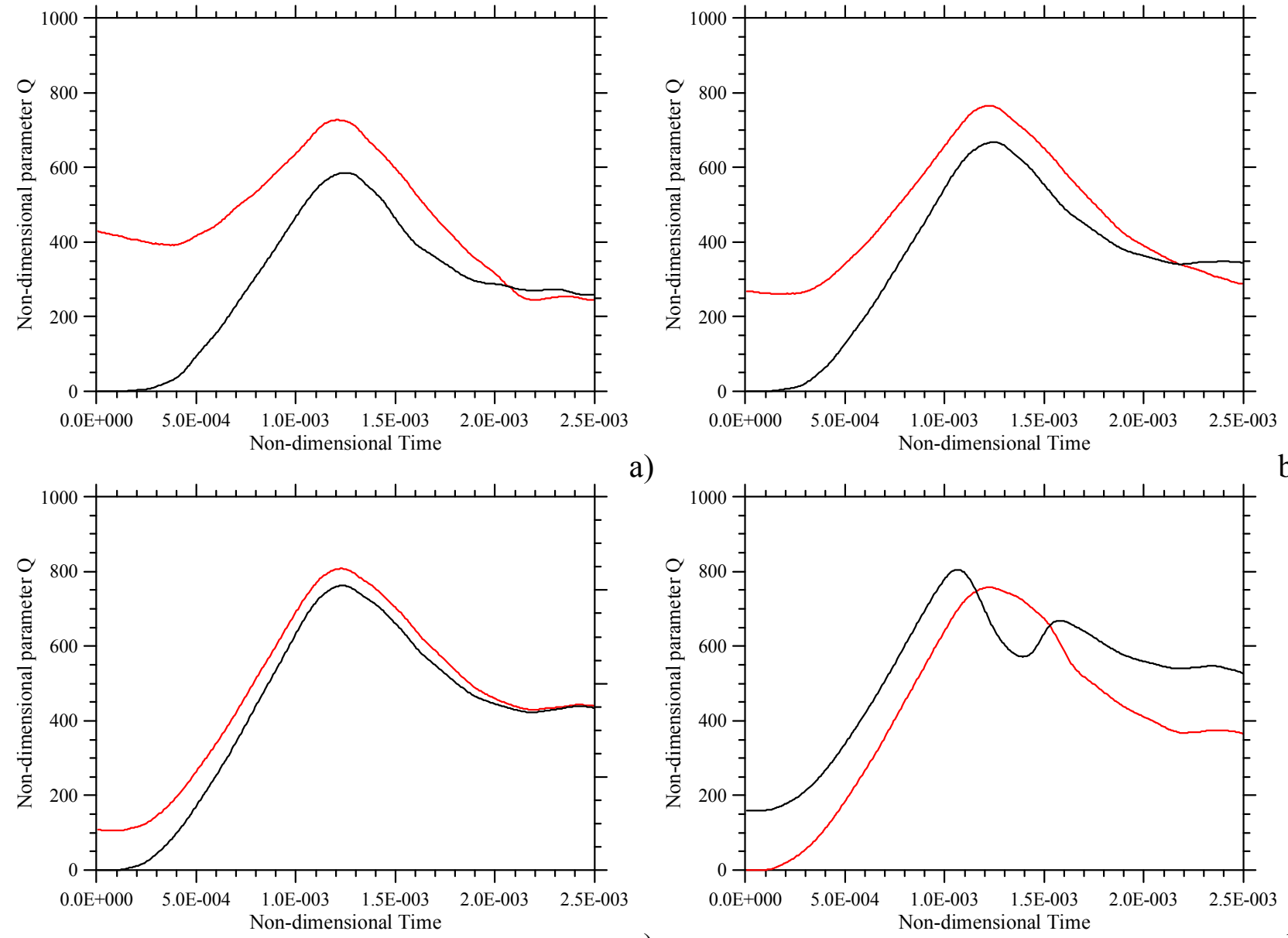

a)

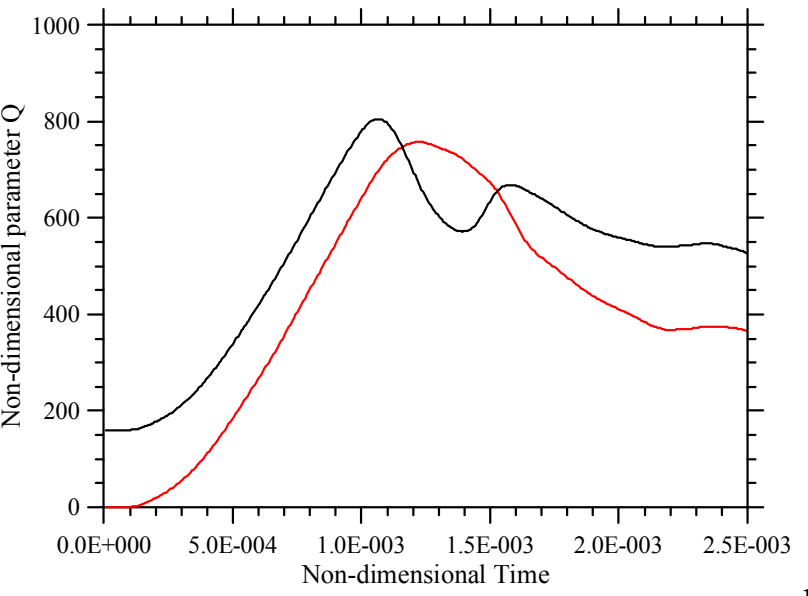

b)

c)
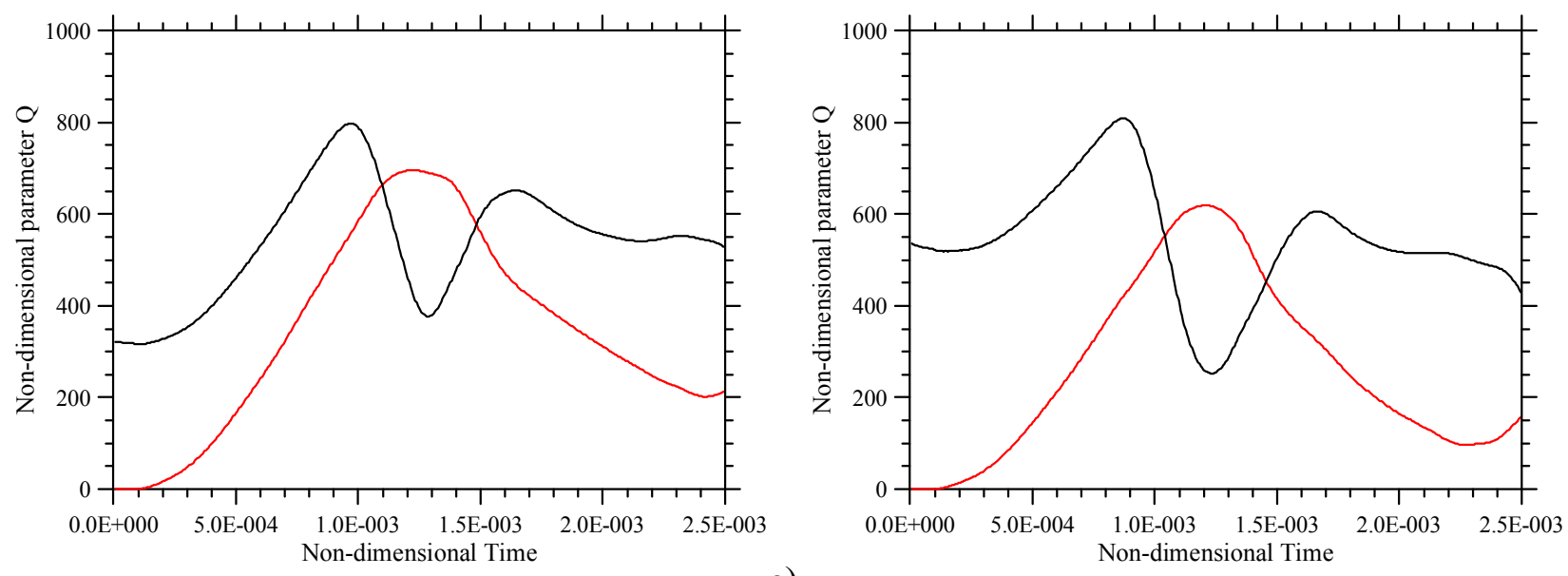

e)

Figure 14: Parameters $Q^{+}$(red line) $Q^{-}$(black line) versus time for $\mathrm{St}=2 \times 10^{-7}, \mathrm{Fr}=5.45 \times 10^{-5}$ $\left(\gamma=2.15 \times 10^{10}\right)$ and different values of the density ratio: a) $\xi=0.2$; b) $\xi=0.5$; c) $\xi=0.8$; d) $\xi=1.3$; e) $\xi=1.6$; f) $\xi=2.0$.

The most interesting feature distinguishing $\xi>1$ from $\xi<1$ cases, however, is the double change in trend (increasing, decreasing, then again increasing and decreasing) observable in the $Q^{-}$plots for 
$\xi>1$ only. This may be seen as a mark of an increased plume ability in influencing the purely gravitational motion of particles for these specific cases (by contrast, no double increasingdecreasing trend can be seen for $\xi<1$ ), where especially in the initial stages of evolution, both particle buoyancy velocities induced on particles by the plume and the gravity field are directed upward. More specifically, the "valley" visible in the central region of the $Q^{-}$plot for $\xi>1$ (the region affected by significant shortage of $Q^{-}$) can be ascribed to the specific stages of evolution taken by the plume in a given temporal neighbourhood of the instant at which it hits the top wall. The location of the minimum of the $Q^{-}$curves for $\xi>1$, indeed, roughly corresponds to this instant. This realisation also leads to two interesting related (less straightforward) arguments, namely, that the two maxima visible on the left and on the right of the valley region (Figs. 14d-f) should be explained on the basis of two extremely different-in-nature effects, these being the not yet fully developed state (in terms of spatial extension and strength) of the plume before it reaches the top wall (see, e.g., Figs. 6a-6e for $\xi=2$ ) and its subsequent weakening (after it has hit the top) due to the creation of two descending layers of fluids at the plume sides (Figs. 6i-6k), and the ensuing (at later times) onset of the (symmetry-breaking) shear-driven instability (Figs. 61-6o).

Figure 14 also shows that, for a fixed St and Fr, apart from the just highlighted trends, the vertical distance (on average) between the two curves is also a function of the density ratio, with such a distance behaving as a decreasing function of $\xi$ for $\xi<1$ and as an increasing function when $\xi>1$.

This information is particularly meaningful as the smaller the separation of the curves, the stronger should be the ability of the plume to retain the particles (maintain particles suspended), which is indeed confirmed by the results shown in Fig. 13 (with the maximum number of suspended particles being attained there for $\xi=0.8$ ).

Similar considerations about the role played by gravity (via Fr) can be obtained by plotting the same quantities $Q^{ \pm}$versus time for a fixed value of $\xi$ and different values of the Stokes and Froude numbers.

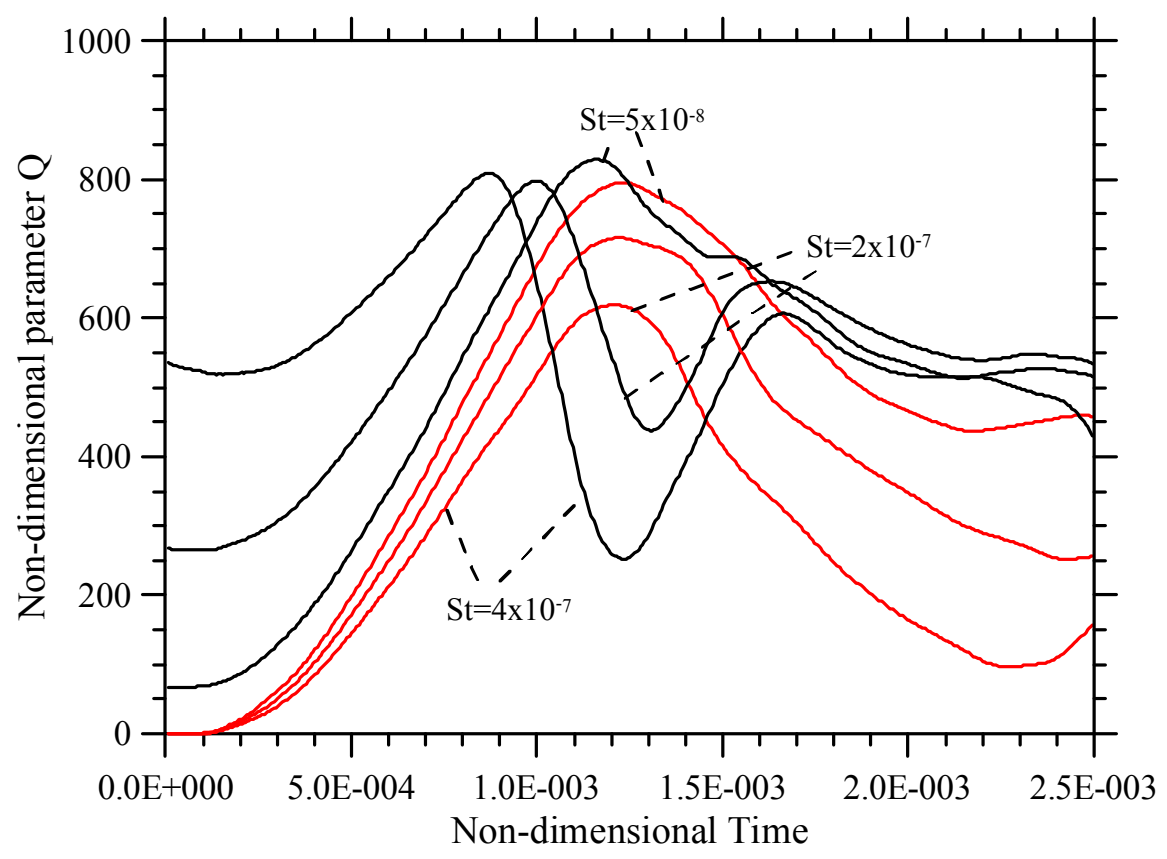




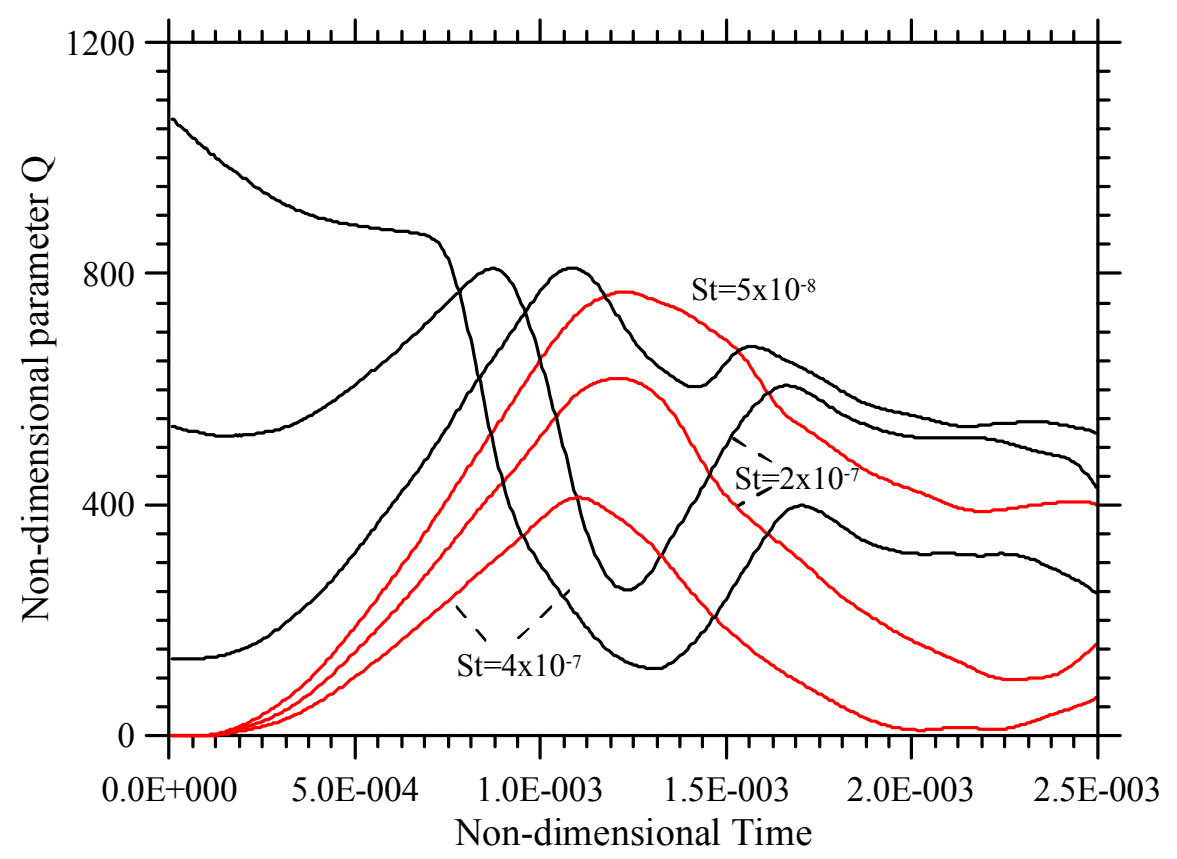

b)

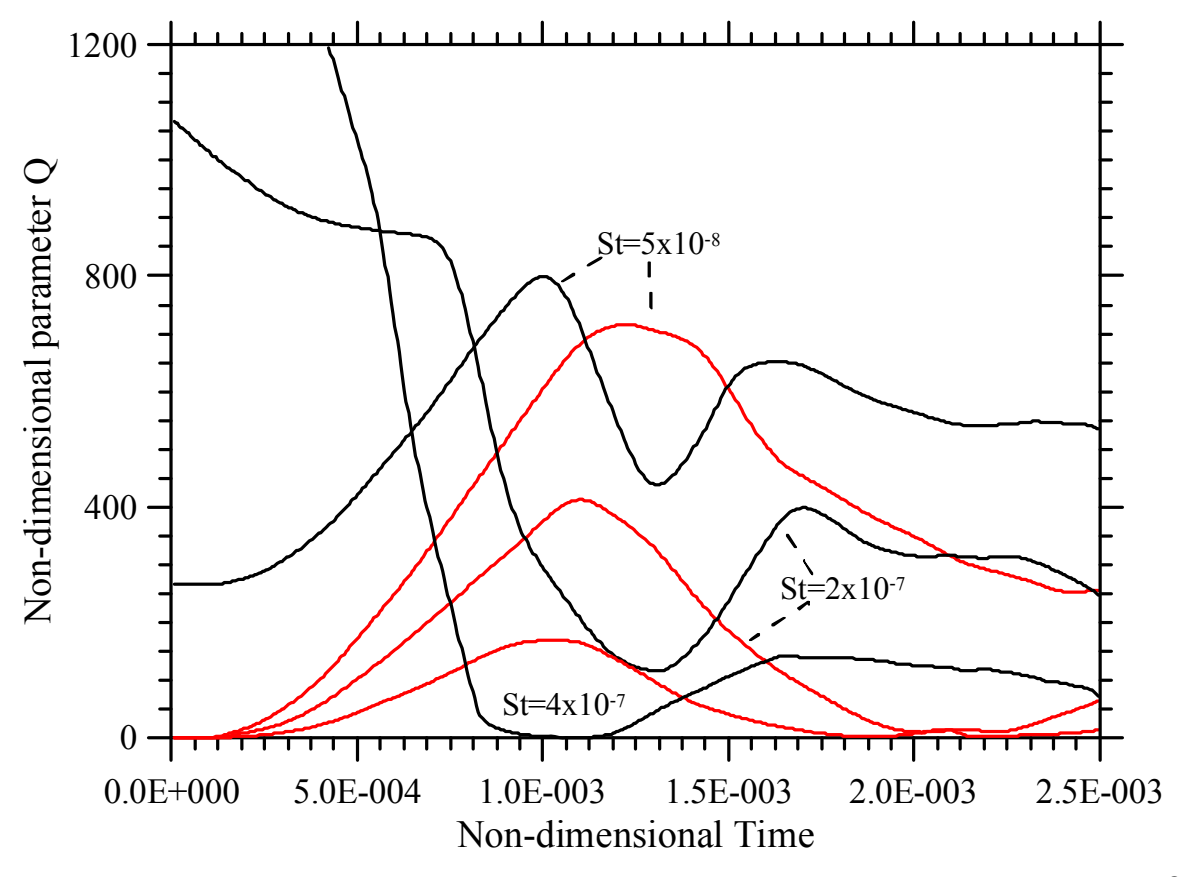

c)

Figure 15: Parameters $Q^{+}$(red line) $Q^{-}$(black line) versus time for $\xi=2.0$, as a function of the Stokes number for different values of the Froude number: a) $\mathrm{Fr}=8 \times 10^{-5}\left(\gamma=1 \times 10^{10}\right)$; b) $\mathrm{Fr}=5.45 \times 10^{-5}$ $\left.\left(\gamma=2.15 \times 10^{10}\right) ; c\right) \mathrm{Fr}=3.85 \times 10^{-5}\left(\gamma=4.3 \times 10^{10}\right)$. 
The related results summarized in Fig. 15 for $\xi=2.0$ allow to identify additional interesting trends. To illustrate them it is convenient to start from the simplest condition possible, namely, the very initial stages of evolution when the plume cap is still confined to the bottom of the container (region located in proximity to $t=0$ in Fig. 15). It can be seen there that $Q^{-}$behaves as an increasing function of St at fixed Fr, and as a decreasing function of Fr at fixed St. These dependences, valid or applicable when the fluid is still in almost quiescent conditions throughout the domain, are clearly in line with what one would expect on the basis of eq. (10) (let us recall that the theoretical sedimentation velocity of particles in a still fluid is directly proportional to the particle Stokes number and inversely proportional to the square of the Froude number).

Extremely interesting in such plots is also the behaviour of the $Q^{+}$curve, which experiences a notable shrinkage in height as the Stokes number is increased for a fixed value of Fr, or as the Froude number is decreased for a fixed value of St.

The first effect can be clearly ascribed to the reduced capacity of heavy particles to be "captured" by the thermal plume vortices when St increases (see the discussions in Sect 2.3).

Let us recall that in the absence of gravity, the two counter-rotating vortices associated with the plume would transport just as many particles from the upper domain into the lower one and vice versa, so that no net settling would take place. In our case, this ideal symmetry is broken by at least two factors: first, gravity itself that cause particle sedimentation, second, the presence of a central rising jet that propels both particles and the two above-mentioned counter-rotating vortices towards the top.

Some particles do not experience significant fluid velocity and settle as they were in a stagnant fluid. Some particles are swept into the return flow and settle at rates that are larger than in still fluid. A limited percentage of them are propelled upward by the strong central jet. Other particles remain suspended, moving on closed trajectories, so that their vertical motions are oscillatory. On the one hand, these different types of behaviour result in a multi-modal distribution of vertical velocities, on the other hand, as a result the mean settling velocity can be either larger or smaller than the terminal velocity in a still fluid.

With these arguments in mind, the dependence of $Q^{+}$on Fr can be properly interpreted recalling what would be the expected theoretical behaviour of the particles in the limit conditions in which $\mathrm{Fr} \rightarrow 0$ (particles undergoing unperturbed purely vertical sedimentation, the reader being referred again to Sect. 3.2).

Additional insights can also be obtained by highlighting that the region of limited temporal extent where the condition $Q^{+}>Q^{-}$is satisfied (clearly identifiable in each of the frames shown in Fig. 15) may be regarded as a further proof of the existence of specific conditions where plume vortices become optimally efficient in promoting heavy particle suspension. 


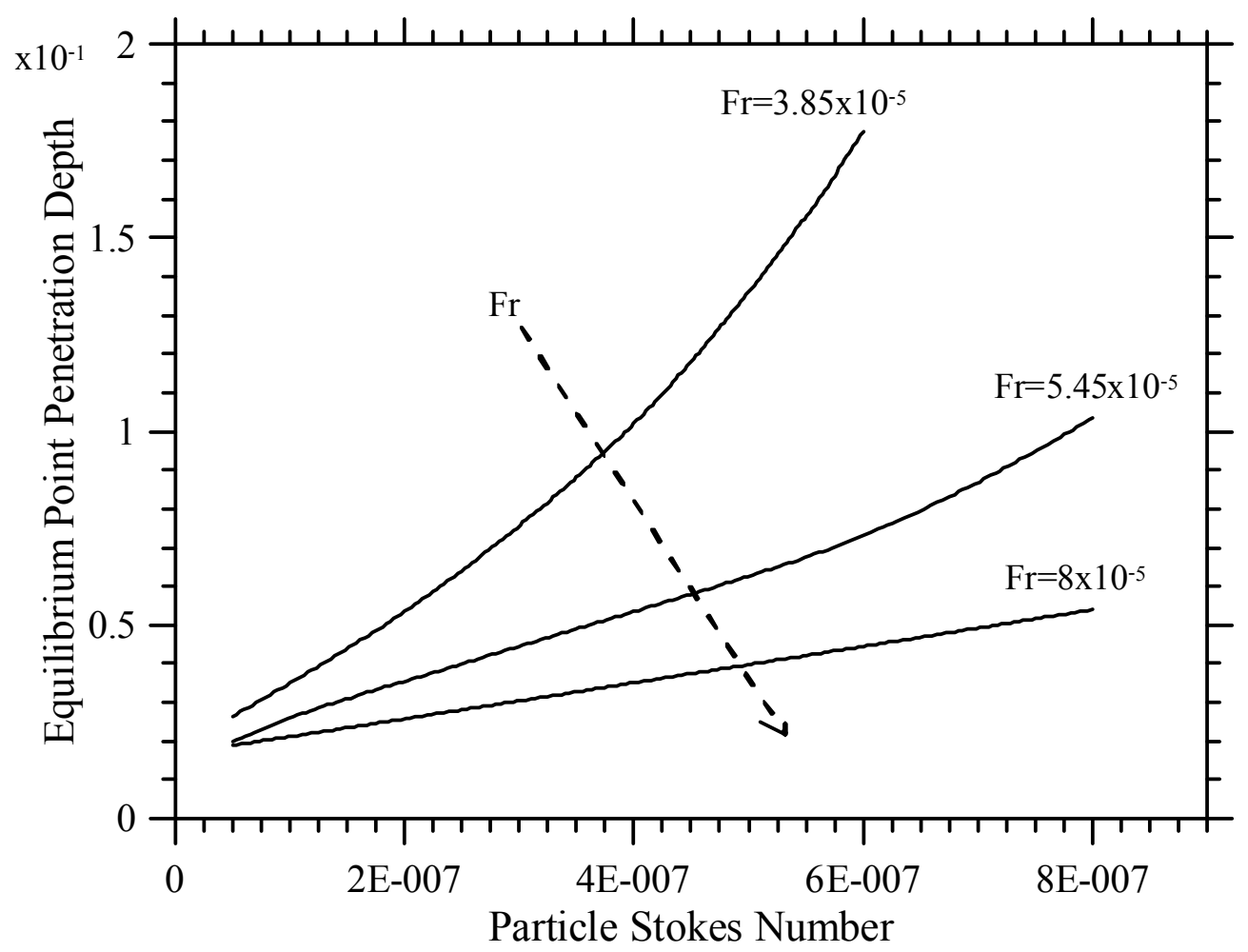

Figure 16: Axial penetration depth of the equilibrium point at $t=4 \times 10^{-4}$ (plume front located at $\mathrm{x}=0.63$ ) for $\xi=2$ as a function of the particle Stokes number for different values of the Froude number.

Figures 16 and 17, finally, provide the missing information about the dependence of the position of the aforementioned dynamically evolving equilibrium point on the system parameters. By taking a look at Fig. 16, the reader will immediately realize that the point penetration depth (distance from plume front) behaves as an increasing function of St, whereas an increase in Fr makes it smaller.

The combined temperature and particle fields in Fig. 17 provide even more interesting details. It is evident there that, for $\mathrm{Fr}=3.85 \times 10^{-5}\left(\gamma=4.3 \times 10^{10}\right)$, a threshold value of the Stokes number can be identified beyond which the existence of the equilibrium point is no longer possible, as witnessed by the notable modification undergone by the particle field in Fig. 17c in terms of topology and morphology of particle path-lines. The topological change is especially evident if one concentrates on the regions located in proximity to the jet core. The typical double $\mathrm{V}$-shaped profiles visible in the particle concentration on both sides (lefty and right) of the jet core (Figs. 17a and 17b) for smaller values of $\mathrm{St}$ are taken over for $\mathrm{St}=6 \times 10^{-7}$ by a new configuration where the two first recognizable path-lines located on the left and on the right of the $\mathrm{x}$ axis proceed almost undisturbed along the vertical direction. Although solid particles flowing through the space encapsulated by these two slightly curved path-lines still experience some deceleration when they pass through the region corresponding to the plume cap, particles are allowed to continue their motion towards the bottom of the domain under the influence of gravity. 

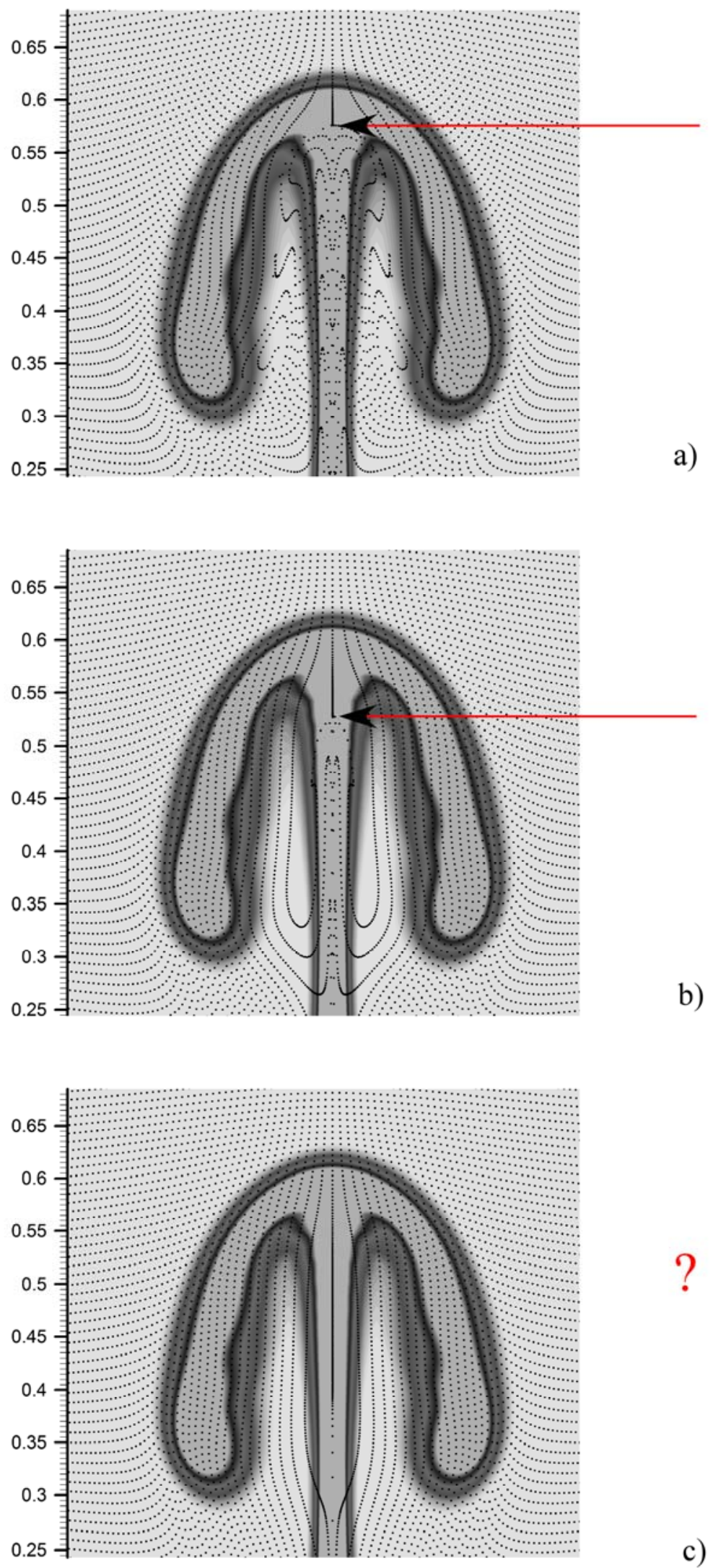

Figure 17: Snapshot of particle distribution and underlying temperature field at $\mathrm{t}=4 \times 10^{-4}$ for $\xi=2$, $\mathrm{Fr}=3.85 \times 10^{-5}\left(\gamma=4.3 \times 10^{10}\right)$ and different values of the particle Stokes number: a) $\mathrm{St}=2 \times 10^{-7}$; b) $\mathrm{St}=4 \times 10^{-7}$; $) \mathrm{St}=6 \times 10^{-7}$. 

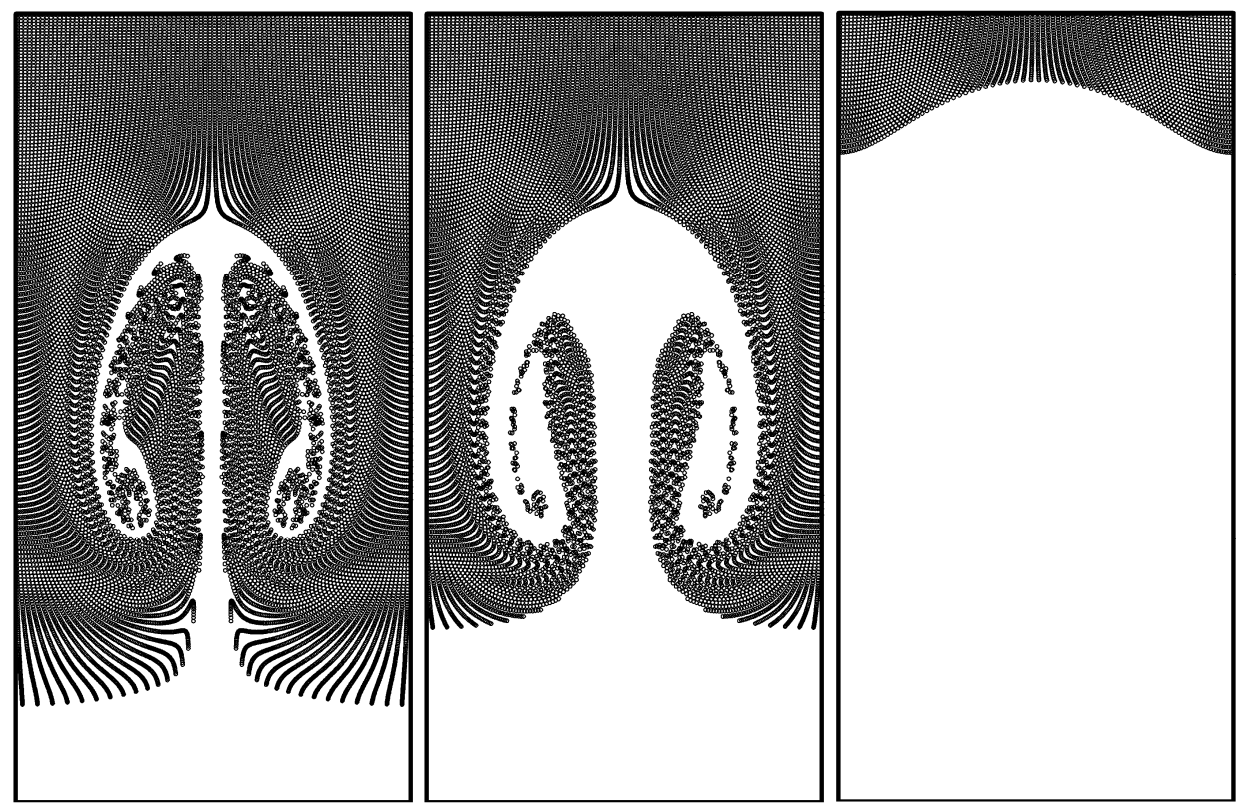

Figure 18: Snapshot of particle distribution at $\mathrm{t}=32\left(\xi=0.5, \mathrm{St}=8 \times 10^{-7}\right)$ for different values of the parameter $\gamma$ : a) $\gamma=1 \times 10^{10}$; b) $\gamma=2.15 \times 10^{10}$; c) $\gamma=4.3 \times 10^{10}$.

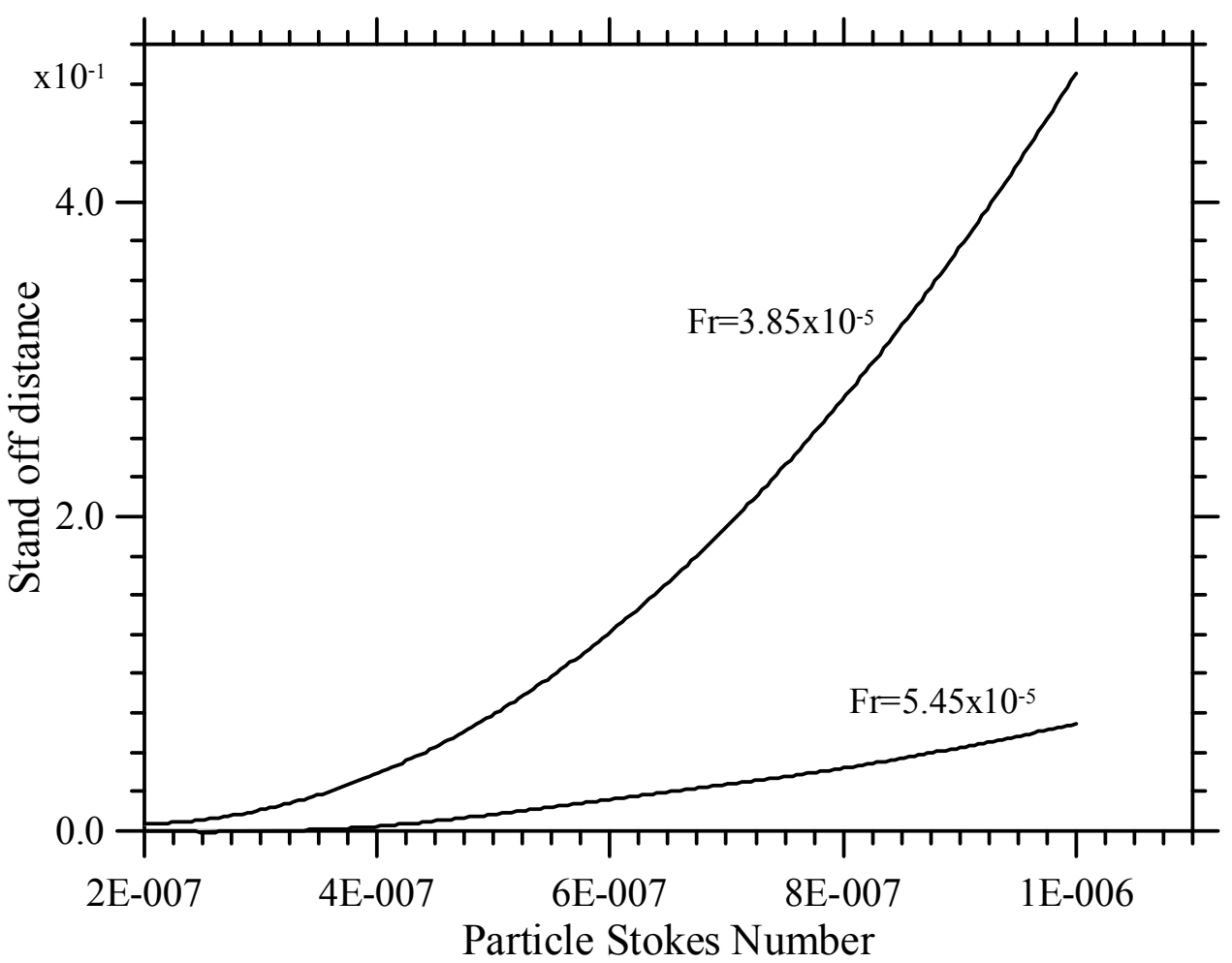

Figure 19: Stand-off distance at $\mathrm{t}=4 \times 10^{-4}$ (plume front located at $\mathrm{x}=0.63$ ) for $\xi=0.5$ as a function of the particle Stokes number for two different values of the Froude number.

Figure 18 provides analogous insights into the influence of the Froude number on the morphology of the separation boundary produced by particles rising for $\xi<1$ under the joint influence of thermal convection and gravity. 
The role particle inertia does still play in such dynamics can be elucidated by plotting the "stand-off distance" (that we define as the distance along the $\mathrm{x}$ direction at $\mathrm{y}=0$ between the plume front and the transition boundary separating the particle dense region from the clear fluid below, see Fig. 11) as a function of St and Fr (Fig. 19). According to such figure, the stronger the particle inertia (as quantified by the Stokes number), the larger the stand-off distance, whereas an increase in the Froude number (corresponding, e.g., to a decrease in the gravity acceleration) has the opposite effect.

\section{Conclusions}

A mathematical and numerical framework has been developed to inquire specifically about the role of thermal convection in determining segregation, dispersion and gravitational sedimentation (or flotation) of solid spherical particles in a convectively unstable medium. In particular, this topic has been investigated by direct numerical solution of the governing flow-field (Navier-StokesBoussinesq) equations in combination with a specific particle tracking method accounting for particle motion under the influence of inertia, viscous drag and gravity. Attention has been paid to a rising plume, which represents a well-known exemplar of localized buoyancy convection.

It has been shown that, if specific conditions are satisfied, particles, initially uniformly spaced in the liquid, can produce recognizable peculiar patterns with well-defined properties varying according to the considered sub-region of the fluid domain, the time interval considered, and particle inertia and drag as quantified by the density ratio and Stokes number and the relative strength of gravity (via the Froude number). Suspension (although non-permanent) is possible in specific regions of the fluid domain, whereas other regions experience rapid depletion processes due to particle sedimentation or flotation. The existence of "equilibrium points" has been investigated as well (together with an analysis of the topological properties displayed by the distribution of suspended particles). All these behaviours have been critically discussed and linked to the general features of the plume (its main constitutive parts and related instabilities). Moreover, to properly track the system evolution, and make results and conclusions sufficiently independent and general, a statistically relevant number of solid particles has been considered. Accordingly, insights into the changing behaviour of the fluidsolid system in the space of parameters have been obtained by connecting the mean evolution of such particles, measured via ad-hoc-defined global parameters $\left(Q^{ \pm}\right)$, to the characteristic problem nondimensional numbers (St, $\xi$ and Fr).

This work should be regarded as a first step towards the understanding of more complex situations. Though our main intention here was to lay out the fundamental dynamical principles governing the interaction of a thermal plume with dispersed solid matter, let us recall once again that thermal plumes are the building blocks of turbulent fluid flow. In such flows, plumes typically stretch, interact with other vortices and undergo a variety of instabilities allowing energy to be transferred to larger scales. Along these lines, among the possible improvements, one direction of future research could be the extension of the present study to the case of turbulent Rayleigh-Bénard convection of which plumes in the IVND regime represent a typical constitutive ingredient. Another possible direction of extension could be the consideration of fluid compressibility effects in the case of 
particles dispersed in gases a very high temperature for which the Boussinesq and other related assumptions are no longer applicable.

\section{References}

[01] Balboa-Usabiaga F., Delgado-Buscalioni R., (2011), Particle hydrodynamics: From molecular to colloidal fluids, Particle-Based Methods II - Fundamentals and Applications, pp. 152-163

[02] Otha M and Sussman M., (2012), The buoyancy-driven motion of a single skirted bubble or drop rising through a viscous liquid, Phys. Fluids, 24(11), 112101.

[03] Zhao J. Z., Ratke L. and Feuerbacher B., (1998), Microstructure evolution of immiscible alloys during cooling through the miscibility gap, Modelling Simul. Mater. Sci. Eng., 6, 123-139.

[04] Zhang Q., Li W., Gu M. and Yanping J., (2006), Dispersion and rheological properties of concentrated silicon aqueous suspension, Powder Technology,161,130-134

[05] Markov A. V., (2008), Thermal conductivity of polymers filled with dispersed particles: A model, Polymer Science Series A, 50(4), 471-479

[06] Zhao J.Z., Ahmed T., Jiang H.X., He J., Sun Q., (2017), Solidification of Immiscible Alloys: A Review, Acta Metallurgica Sinica (English Letters), 30(1), 1-28.

[07] Bürger R. and Wendland W.L., (2001), Sedimentation and suspension flows: Historical perspective and some recent developments, Journal of Engineering Mathematics, 41, 101-116.

[08] Parchevsky K.V., (2000), A new method for the reconstruction of the particle radius distribution function from the sedimentation curve, Chemical Engineering Journal, 80, 73-79.

[09] Parchevsky K.V., (2001), Numerical simulation of sedimentation in the presence of 2D compressible convection and reconstruction of the particle-radius distribution function, Journal of Engineering Mathematics, 41, 203-219.

[10] Reeks M. and Skyrme G., (1976), The dependence of particle deposition velocity on particle inertia in turbulent pipe flow, J. Aerosol Sci., 7, 485-495.

[11] Dasgupta S., Jackson R., Sundaresan S., (1998), Gas-particle flow in vertical pipes with high mass loading of particles, Powder Technology, 96, 6-23.

[12] Maxey M. R., (1987), Gravitational settling of aerosol particles in homogeneous and random flow fields, J. Fluid Mech., 174, 441-465.

[13] Wang L.-P. and Maxey M.R., (1993), Settling velocity and concentration distribution of heavy particles in homogeneous isotropic turbulence, J. Fluid Mech., 256, 27-68.

[14] McGrattan K.B., Baum H.R, and Rehm R.G., (1998), Large eddy simulations of smoke movement, Fire Safety Journal, 30, 161-178.

[15] Kissane M.P., (2008), On the nature of aerosols produced during a severe accident of a watercooled nuclear reactor, Nucl. Eng. Des., 238, 2792-2800.

[16] Moroz B. E., Beck H.L., Bouville A., and Simon S.L., (2013), Predictions of dispersion and fallout from nuclear testing using the NOAA-HYSPLIT meteorological model, Health Phys. 2010 Aug; 99(2): 10.1097/HP.0b013e3181b43697.

[17] Valentine G.A. and Wohletz K.H., (1989), Numerical Models of Plinian Eruption Columns and Pyroclastic Flows, Journal of Geophysical Research, 94(B2), 1867-1887

[18] Haszpra T. and Tél T., (2011), Volcanic ash in the free atmosphere: A dynamical systems approach, Journal of Physics: Conference Series 333, 012008 (12 pages)

[19] Bucchignani E. and Stella F., (1999), Rayleigh-Bénard convection in limited domains: Part 2 transition to chaos, Num. Heat Tr. Part A, 36(1), 17-34.

[20] Lappa M., (2011), Some considerations about the symmetry and evolution of chaotic RayleighBénard convection: The flywheel mechanism and the "wind" of turbulence, Comptes Rendus Mécanique, 339, 563-572. 
[21] Hier Majumder C. A., Yuen D. A. and Vincent A., (2004), Four dynamical regimes for a starting plume model, Phys. Fluids, 16(5), 1516-1531.

[22] Melnikov D.E., Pushkin D.O., Shevtsova V.M., (2013), Synchronization of finite-size particles by a traveling wave in a cylindrical flow, Phys Fluids, 25 (9), 092108

[23] Melnikov D.E. and Shevtsova V., (2017), Different types of Lagrangian coherent structures formed by solid particles in three-dimensional time-periodic flows, European Physical Journal: Special Topics, 226(6), 1239-1251.

[24] Lappa M., (2016), On the nature, formation and diversity of particulate coherent structures in Microgravity Conditions and their relevance to materials science and problems of Astrophysical interest, Geophysical and Astrophysical Fluid Dynamics, 110(4), 348-386.

[25] Maxey M.R., Patel B.K., Chang E.J., Wang L.-P., (1997), Simulations of dispersed turbulent multiphase flow, Fluid Dyn. Res., 20(1-6), 143-156.

[26] Saw E. W., Shaw R. A., Ayyalasomayajula S., Chuang P. Y., and Gylfason A., (2008), Inertial Clustering of Particles in High-Reynolds-Number Turbulence, Phys. Rev. Lett., 100, 214501.

[27] Boffetta G. and De Lillo F., (2004), Large scale inhomogeneity of inertial particles in turbulent flows, Phys. Fluids, 16(4), L20-L23

[28] Squires K. D. and Eaton J.K., (1991), Preferential concentration of particles by turbulence, Phys. Fluids 3, 1169-1178.

[29] Biferale L., Moffetta G., Celani A., Lanotte A., Toschi F., (2005), Particle trapping in threedimensional fully developed turbulence, Phys. Fluids, 17, 021701 (2005), 4 pages

[30] Bec J., Biferale L., Cencini M., Lanotte A., Musacchio S., and Toschi F., (2007), Heavy Particle Concentration in Turbulence at Dissipative and Inertial Scales, Phys. Rev. Lett., 98, 084502.

[31] Eaton J.K. and Fessler J.R., (1994), Preferential concentration of particles by turbulence, Int. J. Multiphase Flow, 20, 169-209.

[32] Raju N. and Meiburg E., (1995), The accumulation and dispersion of heavy particles in forced two-dimensional mixing layers. Part 2: The effect of gravity, Phys. Fluids, 7, 1241-1264.

[33] Stommel H., (1949), Trajectories of small bodies sinking slowly through convection cell, $J$. Marine Res., 8, 24.

[34] Maxey M. R. and Corrsin S., (1986), Gravitational settling of aerosol particles in randomly oriented cellular flow fields, J. Atmos. Sci., 43, 1112-1134.

[35] Clift R., Grace J.R., Weber M.E. , (1978), Bubbles, drops and particles, Academic Press, New York, 1978.

[36] Raju N. and Meiburg E., (1997), Dynamics of small spherical particles in vortical and stagnation point flow fields, Phys. Fluids, 9, 299-314.

[37] Dávila J. and Hunt J. C. R., (2001), Settling of small particles near vortices and in turbulence, J. Fluid Mech., 440, 117-145.

[38] Eames I. and Gilbertson M. A., (2004), The settling and dispersion of small dense particles by spherical vortices, J. Fluid Mech., 498, 183- 203

[39] Chen L., Goto S. and Vassilicos J. C., (2006), Turbulent clustering of stagnation points and inertial particles, J. Fluid Mech., 553, 143-154.

[40] Ravichandran S., Perlekar P. and Govindarajan R., (2014), Attracting fixed points for heavy particles in the vicinity of a vortex pair, Phys. Fluids, 26, 013303.

[41] Bergougnoux L., Bouchet G., Lopez D. and Guazzelli E., (2014), The motion of solid spherical particles falling in a cellular flow field at low Stokes number, Phys. Fluids 26, 093302.

[42] Good G. H., Ireland P. J., Bewley G. P., Bodenschatz E., Collins L. R. and Warhaft Z., (2014), Settling regimes of inertial particles in isotropic turbulence, J. Fluid Mech., 759, R3.

[43] Kawanisi K. and Shiozaki R., (2008), Turbulent effects on the settling velocity of suspended sediment, J. Hydraul. Engng 134 (2), 261-266.

[44] Akbar M.K., Rahman M., Ghiaasiaan S.M., (2009), Particle transport in a small square enclosure in laminar natural convection, Aerosol Sci., 40, 747-761. 
[45] Puragliesi R., Dehbi A., Leriche E., Soldati A., Deville M., (2011), DNS of buoyancy-driven flows and lagrangian particle tracking in a square cavity at high Rayleigh numbers. Int. J. Heat Fluid Flow, 32, 915-931.

[46] Lappa M., Thermal Convection: Patterns, Evolution and Stability (John Wiley \& Sons, Chichester, England, 2009).

[47] Lappa M., (2013), On the variety of particle accumulation structures under the effect of gjitters, J. Fluid Mech., 726, 160-195.

[48] Lappa M., (2013), Assessment of the role of axial vorticity in the formation of Particle Accumulation Structures (PAS) in supercritical Marangoni and hybrid thermocapillary-rotationdriven flows, Phys. Fluids, 25(1) 012101 (11 pages)

[49] Lappa M., (2013), On the Existence and Multiplicity of One-dimensional Solid Particle Attractors in Time-dependent Rayleigh-Bénard Convection, Chaos, 23(1), 013105 (9 pages).

[50] Lappa M., (2014), Stationary Solid Particle Attractors in Standing Waves, Phys. Fluids, 26(1), 013305 (12 pages)

[51] Lappa M., (2014), The patterning behaviour and accumulation of spherical particles in a vibrated non-isothermal liquid, Phys. Fluids, 26(9), 093301 (22 pages).

[52] Lappa M., (2016), Numerical study into the morphology and formation mechanisms of threedimensional particle structures in vibrated cylindrical cavities with various heating conditions, Physical Review Fluids, 1(6), 064203 (25 pages).

[53] Lappa M., (2017), On the multiplicity and symmetry of particle attractors in confined nonisothermal fluids subjected to inclined vibrations, Int. J. Multiphase Flow, 93, 71-83.

[54] Gan H., Chang J., Feng J.J. and Hu H.H., (2003), Direct numerical simulation of the sedimentation of solid particles with thermal convection, J. Fluid Mech., 481, 385-411.

[55] Haeri S. and Shrimpton J.S., (2014), Fully resolved simulation of particle deposition and heat transfer in a differentially heated cavity, Int. J. Heat Fluid Flow, 50, 1-15.

[56] Rouse H., Yih C.S., and Humphreys H.W., (1952), Gravitational convection from a boundary source, Tellus, 4, 201-210.

[57] Batchelor G.K., (1954), Heat convection and buoyancy effects in fluids, Q. J. R. Met. Soc. 80, 339-358.

[58] Gebhart B., (1962), Effects of viscous dissipation in natural convection, J. Fluid Mech., 14, 225232

[59] Worster M. G., (1986), The axisymmetric laminar plume: asymptotic solution for large Prandtl number, Stud. Appl. Maths, 75, 139-152.

[60] Liñán A. and Kurdyumov V.N., (1998), Laminar free convection induced by a line heat source, and heat transfer from wires at small Grashof numbers, J. Fluid Mech., 362, 199-227.

[61] Moses E., Zocchi G., and Libchaber A., (1993), An experimental study of laminar plumes, J. Fluid Mech., 251, 581-601.

[62] Kaminski E. and Jaupart C., (2003), Laminar starting plumes in high-Prandtl-number fluids, J. Fluid Mech, 478, 287-298.

[63] Fujii T., (1963), Theory of the steady laminar natural convection above a horizontal line source and a point heat source, Int. J. Heat Mass Transfer, 16, 597-606.

[64] Brand R. and Lahey F., (1967), The heated laminar vertical jet, J. Fluid Mech., 29, 305-315.

[65] Hernandez R.H., (2015), Natural convection in thermal plumes emerging from a single heat source, Int. J. Thermal Sciences 98, 81-89.

[66] Marble F. E., (1970), Dynamics of dusty gases, Ann. Rev. Fluid Mech., 2, 397-446.

[67] De' Michieli Vitturi M., Esposti Ongaro T., Neri A., Salvetti M.V., Beux F., (2007), An immersed boundary method for compressible multiphase flows: application to the dynamics of pyroclastic density currents, Comput. Geosci., 11, 183-198.

[68] Maxey M. R. and Riley J. J., (1983), Equation of motion for a small rigid sphere in a nonuniform flow, Phys. Fluids, 26, 883-889. 
[69] Sapsis T. and Haller G., (2010), Clustering criterion for inertial particles in two-dimensional time-periodic and three-dimensional steady flows, Chaos, 20, 017515.

[70] Kuhlmann H.C.et al., (2014), The JEREMI-Project on thermocapillary convection in liquid bridges. Part A: Overview of Particle Accumulation Structures, Fluid Dyn. Mater. Process., 10 (1), $1-36$.

[71] Ladyzhenskaya O.A., (1969), The Mathematical Theory of Viscous Incompressible Flow, Gordon and Breach, 2nd Edition, New York - London, 1969.

[72] Lappa M. and Gradiscak T., (2018), On the Oscillatory Modes of Compressible Thermal Convection in inclined differentially heated cavities, Int. J. of Heat and Mass Transfer, 121, 412-436. [73] Paolucci S., (1990), Direct numerical simulation of two-dimensional turbulent natural convection in an enclosed cavity, J. Fluid Mech., 215, 229-262.

[74] Farhangnia M., Biringen S, Peltier L. J., (1996), Numerical Simulation of Two-dimensional Buoyancy-driven Turbulence in a Tall Rectangular Cavity, Int. J. Numer. Meth. Fluids, 23(12), 1311 $-1326$.

[75] Kerr R.M., (1996), Rayleigh number scaling in numerical convection, J. Fluid Mech. 310, 139179.

[76] De A.K., Eswaran V., Mishra P.K., (2017), Scalings of heat transport and energy spectra of turbulent Rayleigh-Bénard convection in a large-aspect-ratio box, Int. J. Heat Fluid Flow, 67, 111124.

[77] Shishkina O., Stevens R.J.A.M., Grossmann S. and Lohse D., (2010), Boundary layer structure in turbulent thermal convection and its consequences for the required numerical resolution, New $J$. Phys. 12, 075022 (17 pages).

[78] Lappa M., (2016), Patterning behaviour of gravitationally modulated supercritical Marangoni flow in liquid layers, Phys. Rev. E Statistical Nonlinear, and Soft Matter Physics, 93(5), 053107, (13 pages).

[79] Lappa M., Drikakis D., Kokkinakis I., (2017), On the propagation and multiple reflections of a blast wave travelling through a dusty gas in a closed box, Phys. Fluids, 29(3), 033301 (19 pages).

[80] Kaminski E., Tait S. and Carazzo G., (2005), Turbulent entrainment in jets with arbitrary buoyancy, J. Fluid Mech., 526, 361-376.

[81] Lee J. H.W. and Asce F., (2012), Mixing of Multiple Buoyant Jets, J. Hydraul. Eng., 138(12), $1008-1021$.

[82] Isaac K.M. and Jakubouski A. K., (1985), Experimental study of the interaction of multiple jets with a cross flow, AIAA Journal, 23(11), 1679-1683.

[83] Desrayaud G. and Lauriat G., (1993), Unsteady confined buoyant plumes, J. Fluid Mech., 252, 617-646

[84] Noto, K. (1989), Swaying motion in thermal plume above a horizontal line heat source, $J$. Thermophys. Heat Transfer , 3 (4), 428-434.

[85] Cortese T. and Balachandar S., (1993), Vortical nature of thermal plumes in turbulent convection, Phys. Fluids A, 5, 3226-3232. 